\title{
RATIONAL NORMAL SCROLLS AND THE DEFINING EQUATIONS OF REES ALGEBRAS
}

\begin{abstract}
Andrew R. Kustin ${ }^{1}$, Claudia Polini ${ }^{2}$, and Bernd F. Ulrich ${ }^{3}$
Abstract. Consider a height two ideal, $I$, which is minimally generated by $m$ homogeneous forms of degree $d$ in the polynomial ring $R=k[x, y]$. Suppose that one column in the homogeneous presenting matrix $\varphi$ of $I$ has entries of degree $n$ and all of the other entries of $\varphi$ are linear. We identify an explicit generating set for the ideal $\mathcal{A}$ which defines the Rees ring $\mathcal{R}=R[I t]$; so $\mathcal{R}=S / \mathcal{A}$ for the polynomial ring $S=R\left[T_{1}, \ldots, T_{m}\right]$. We resolve $\mathcal{R}$ as an $S$-module and $I^{S}$ as an $R$-module, for all powers $s$. The proof uses a rational normal scroll $\operatorname{ring} A=S / H$ with $\mathcal{A} A$ isomorphic to the $n^{\text {th }}$ symbolic power of a height one prime ideal $K$ of $A$. The ideal $K^{(n)}$ is generated by monomials. Whenever possible, we study $A / K^{(n)}$ in place of $A / \mathcal{A} A$ because the generators of $K^{(n)}$ are much less complicated then the generators of $\mathcal{A} A$. We obtain a filtration of $K^{(n)}$ in which the factors are polynomial rings, hypersurface rings, or modules resolved by Eagon-Northcott complexes. The generators of $I$ give rise to an algebraic curve $\mathcal{C}$ in projective $m-1$ space. The defining equations of the fiber ring $\mathcal{R} /(x, y) \mathcal{R}$ yield a solution of the implicitization problem for $\mathcal{C}$.
\end{abstract}

\section{Introduction.}

Let $I$ be a height two ideal in the polynomial ring $R=k[x, y]$, with $I$ minimally generated by $m$ forms of degree $d$. We consider the following questions. What is the defining ideal, $\mathcal{A}$, of the Rees ring

$$
\mathcal{R}=R[I t]=R \oplus I t \oplus I^{2} t^{2} \oplus \cdots ?
$$

What is the Hilbert function of $\mathcal{R}$ ? The Rees ring $\mathcal{R}$ is equal to $R\left[T_{1}, \ldots, T_{m}\right] / \mathcal{A}$, what is the minimal resolution of $\mathcal{R}$ ? What are the defining equations of the fiber

\footnotetext{
${ }^{1}$ Supported in part by the National Security Agency.

${ }^{2}$ Supported in part by the National Science Foundation and the National Security Agency.

${ }^{3}$ Supported in part by the National Science Foundation.

2000 Mathematics Subject Classification. Primary: 14Q05, Secondary: 13C20, 13D02, 14E05, 14M12, 68W30.

Key words and phrases. Divisorial Ideal, Eagon-Northcott Complex, Fiber Ring, Hilbert-Burch Theorem, Implicitization, Rational Normal Scroll, Rees Algebra.
} 
ring $\mathcal{F}=k \otimes_{R} \mathcal{R}$ ? What is the Hilbert function of $\mathcal{F}$ ? What is the minimal resolution of $\mathcal{F}=k\left[T_{1}, \ldots, T_{m}\right] / \mathcal{A} k\left[T_{1}, \ldots, T_{m}\right]$ ? What is the Hilbert function of $R / I^{s}$ and what is the minimal resolution of $R / I^{s}$, for all powers $s$ ?

The question about the defining equations of the Fiber ring of $I$ is of particular interest to the geometric modeling community. The ideal $I=\left(\delta_{1}, \ldots, \delta_{m}\right)$ gives rise to an algebraic curve by way of the rational map $\phi: \mathbb{P}^{1} \rightarrow \mathbb{P}^{m-1}$ which sends $\left(x_{0}, y_{0}\right)$ to $\left(\delta_{1}\left(x_{0}, y_{0}\right), \ldots, \delta_{m}\left(x_{0}, y_{0}\right)\right)$. The closed image of $\phi$ is an algebraic curve $\mathcal{C}$. The computation of the defining equations of the fiber ring $\mathcal{F}$ is called the implicitization problem for the curve $\mathcal{C}$; see, for example, $[3,5,6,13]$. One technique for attacking the implicitization problem for $\mathcal{C}$ is to use the method of moving curves $[8,7,4]$. To an algebraist, the moving curve ideal of $\phi$ is the defining ideal, $\mathcal{A}$, of the Rees ring of $I$. The Rees ring $\mathcal{R}$ Rees ring encodes many of the analytic properties of the variety defined by $I$. It provides an algebraic realization for the classical notion of blowingup a variety along a subvariety, and plays an important role in the birational study of algebraic varieties, particularly in the study of desingularization.

The Hilbert Burch Theorem guarantees that the ideal $I$ is generated by the maximal order minors of an $m \times(m-1)$ matrix $\varphi$ with homogeneous entries. In addition to $m$ and $d$ the other important piece of data is the column degrees of $\varphi$. In the present paper, the column degrees of $\varphi$ are $(1, \ldots, 1, n)$. In other words, the entries of one column of $\varphi$ have arbitrary degree $n$, all of the other entries of $\varphi$ are linear. We identify the generators of $\mathcal{A}$ in Theorem 3.6. We resolve $I^{s}$ in Theorems 6.1 .

In the proof of Theorem 1.11, we identify an ideal $H$ of $S=R\left[T_{1}, \ldots, T_{m}\right]$ with $A=S / H$ a normal domain and $\mathcal{A} A$ a height one ideal of $A$. The ideal $\mathcal{A} A$ is necessarily prime; hence, $\mathcal{A} A$ is a divisorial ideal of $A$. The ring $A$ is the coordinate ring of a rational normal scroll; the divisor class group of such rings is studied in [22]. We identify a prime ideal $K$ in $A$ so that the $n^{\text {th }}$ symbolic power, $K^{(n)}$, of $K$ is isomorphic to $\mathcal{A} A$. The ideal $K^{(n)}$ in $A$ is generated by monomials. We record an explicit isomorphism $K^{(n)} \rightarrow \mathcal{A} A$ and an explicit generating set for $\mathcal{A} A$. The idea of looking for an uncomplicated divisorial ideal in a normal domain $S / H$ was inspired by our reading of [16] where the case $m=3$ is studied: we reinterpreted the Sylvester forms of [16] as isomorphisms of divisorial ideals in the hypersurface ring defined by $x T_{1}-y T_{2}$.

The generating set for $\mathcal{A} A$ that we calculate in section three is very explicit, but fairly complicated. We use the isomorphic model $K^{(n)}$, which is generated by monomials, rather than the explicit generators of $\mathcal{A} A$, to make the rest of our calculations. We compute the Hilbert function of $\mathcal{R}$, the Hilbert function of $I^{s}$, the resolution of $I^{s}$, and the resolution of $\mathcal{R}$, all using essentially one trick. That is, we refine the filtration $0 \subseteq \mathcal{A} A \subseteq A$ in such a way that the corresponding refinement of $0 \subseteq K^{(n)} \subseteq A$ has factors that are easy for us to study; these factors are 
Eagon-Northcott modules (in the sense that they are resolved by Eagon-Northcott complexes).

For the time being we return to the general case where the column degrees of $\varphi$ are arbitrary, rather than $(1, \ldots, 1, n)$. When one studies the questions of the first paragraph it is important to know if the map $\phi: \mathbb{P}^{1} \rightarrow \mathbb{P}^{m-1}$ is birational. Indeed, some hypothesis which is equivalent to " $\phi$ is birational" appears in many of the results of [16] and [4]. In [23] we reprove Hurwitz's Theorem [14, Chapt. IV, sect. 2] in the present context. Let $r$ be the degree of the map $\phi: \mathbb{P}^{1} \rightarrow \mathcal{C}$; so $r$ is also equal to the dimension of the field extension $\operatorname{Quot}(\mathcal{F}) \subseteq \operatorname{Quot}\left(R^{(d)}\right)$, where "Quot" means quotient field and $R^{(d)}$ is the $d^{\text {th }}$ Veronese ring associated to $R$. We prove that there exist homogeneous forms $f_{1}, f_{2}$ of degree $r$ in $R$ such that a generating set of $I$ actually lives in the polynomial ring $k\left[f_{1}, f_{2}\right]$. Thus, the non-birational case is the same as the birational case with a new set of variables - results which hold in the birational case may be extended to the non-birational case in a straightforward manner. Also, if the map $\phi$ is not birational, then the number $r$ must divide each column degree in the matrix $\varphi$; consequently, if the column degrees of $\varphi$ are relatively prime (as they are in the present paper), then the map $\phi: \mathbb{P}^{1} \rightarrow \mathbb{P}^{m-1}$ is automatically birational. The paper [23] also contains a number of results which hold under the hypothesis that at least one column of $\varphi$ is linear. These results apply in the situation of the present paper and they include a proof that the Rees algebra $\mathcal{R}$ satisfies the regularity condition $R_{1}$, a calculation of the canonical module of $\mathcal{R}$, and a calculation of the core of $I$.

In $[24]$ we consider the questions of the first paragraph when none of the columns of $\varphi$ are linear. In this case we have not found a suitable candidate for $H$ and an uncomplicated divisorial ideal in $S / H$ which is isomorphic to $\mathcal{A} / H$. Instead, our approach is based on ideas about Morley forms as found in $[4,19,20]$.

Return now to the ambient hypothesis of the present paper. In other words, assume that the column degrees of the presenting matrix $\varphi$ are $(1, \ldots, 1, n)$. It is shown in $[10,21,28]$ that the Castelnuovo-Mumford regularity of $I^{s}$ is a linear function of $s$ for all $s \gg 0$. Indeed, in our notation, the aforementioned papers guarantee that $\operatorname{reg}\left(I^{s}\right)=s d+e$ for some non-negative integer $e$ and all $s \geq s_{0}$, for some $s_{0}$. We have resolved $I^{s}$, for all $s$; so, we are able to read the exact value of $e$ and to determine the least value of $s_{0}$ for which the above equation holds. The answers are much different depending on the minimal number of generators of the row space of the linear part, $\varphi^{\prime}$, of $\varphi$. When the row space of $\varphi^{\prime}$ can be generated by fewer than $m$ generators, then $e=n-1$ and $s_{0}=1$. On the other hand, when the row space of $\varphi^{\prime}$ requires $m$ generators, then $e=0$ and $s_{0}=\left\lceil\frac{n-1}{\sigma_{2}}\right\rceil+1$, where $\sigma_{1} \geq \sigma_{2}$ is the partition of $m-2$ that corresponds to the canonical form of $\varphi^{\prime}$. A recent theorem of Eisenbud and Harris [12]) interprets the value of $e$.

In section one we establish the connection between the Rees algebra $\mathcal{R}(I)$ and a 
rational normal scroll ring. We also list the generators of the ideal $K^{(n)}$ which is isomorphic to the defining ideal of $\mathcal{R}(I)$. In section two we find a canonical form for the linear part of the matrix which presents the ideal $I$. The form is used in section one; furthermore, this form allows us to calculate the regularity of $I^{s}$ for all $s$. We record an explicit generating set for the defining ideal of $\mathcal{R}(I)$ in section three. In section four we calculate the reduction number of $I$, the regularity and depth of $\mathcal{F}(I)$, and the depth $\mathcal{R}(I)$. The filtration of $K^{(n)}$ by Eagon-Northcott modules is in section five. In section six we resolve $I^{s}$ and verify the regularity calculation of section two.

In this discussion $R$ is a standard graded polynomial ring over a field. If $N$ is a finitely generated non-zero graded $R$-module and

$$
0 \rightarrow F_{k} \rightarrow \cdots \rightarrow F_{0} \rightarrow N \rightarrow 0
$$

with $F_{i}=\bigoplus_{j=1}^{\beta_{i}} R\left(-t_{i, j}\right)$, is the minimal homogeneous resolution of $N$ by free $R$-modules, then the Castelnuovo-Mumford regularity of $N$ is equal to $\operatorname{reg}(N)=$ $\max _{i, j}\left\{t_{i, j}-i\right\}$.

Convention. Throughout this paper, $k$ is a field; every ring $A$ that we consider is graded and finitely generated as an algebra over $A_{0}=k$; and every $A$-module $M=\bigoplus M_{i}$ that we consider is graded and finitely generated. We use $\lambda_{A}\left(\_\right)$for the length of an $A$-module. It follows that

$$
\lambda_{k}\left(M_{i}\right), \quad \lambda_{A}\left(\frac{\bigoplus_{i \leq j} M_{j}}{\bigoplus_{i<j} M_{j}}\right), \quad \text { and } \quad \operatorname{dim}_{k}\left(M_{i}\right)
$$

are equal. We write $\lambda\left(M_{i}\right)$ for the common value. Of course, all three numbers are equal to the value of the Hilbert function $\mathrm{H}_{M}$ at $i$, denoted $\mathrm{H}_{M}(i)$.

Convention. For each statement "S", we define

$$
\chi(S)= \begin{cases}1, & \text { if } \mathrm{S} \text { is true, and } \\ 0, & \text { if } \mathrm{S} \text { is false }\end{cases}
$$

In particular, $\chi(i=j)$ has the same value as the Kronecker delta $\delta_{i j}$.

Notation. If $\theta$ is a real number, then $\lceil\theta\rceil$ and $\lfloor\theta\rfloor$ are the "round up" and "round down" of $\theta$, respectively; that is, $\lceil\theta\rceil$ and $\lfloor\theta\rfloor$ are the integers with

$$
\lceil\theta\rceil-1<\theta \leq\lceil\theta\rceil \text { and }\lfloor\theta\rfloor \leq \theta<\lfloor\theta\rfloor+1 .
$$


The graded ring $S=\bigoplus_{i \geq 0} S_{i}$ is a standard graded $S_{0}$-algebra if $S$ is generated as an $S_{0}$-algebra by $S_{1}$ and $S_{1}$ is finitely generated as an $S_{0}$-module. For any terms or concepts that we neglected to define, consult [1] first.

\section{Rees algebras and rational normal scrolls.}

Let $k$ be a field, $R$ be the standard graded polynomial $\operatorname{ring} R=k[x, y]$, and $I$ be a height two ideal of $R$ which is minimally generated by $m$ homogeneous forms of degree $d$. The Hilbert-Burch Theorem guarantees that $I$ is generated by the maximal order minors of an $m \times(m-1)$ matrix $\varphi$, with homogeneous entries. In this paper, the entries of one column of $\varphi$ have degree $n$; all of the other entries of $\varphi$ are linear. So, $d=n+m-2$ and the resolution of $I$ looks like

Goal

$$
0 \rightarrow \begin{gathered}
R(-d-1)^{m-2} \\
R(-d-n)
\end{gathered} \stackrel{\varphi}{\rightarrow} R(-d)^{m} \stackrel{\left[\begin{array}{lll}
\delta_{1} & \ldots & \delta_{m}
\end{array}\right]}{\longrightarrow} I \rightarrow 0
$$

The Rees algebra of $I$ is equal to $\mathcal{R}(I)=R[I t]$. Let $S$ be the polynomial ring $S=R\left[T_{1}, \ldots, T_{m}\right]$ in $m$ indeterminates over $R$ and let $\mathcal{A}$ be the kernel of the $R$ algebra homomorphism $\Phi: S \rightarrow \mathcal{R}(I)$ which sends $T_{i}$ to $\delta_{i} t$. In this section we identify a normal domain $A=S / H$ so that $A$ is the coordinate ring of a rational normal scroll and $\mathcal{A} A$ is a height one ideal of $A$. We also identify an explicit divisorial ideal $K^{(n)}$ of $A$ which is generated by monomials and an explicit element $g$ of $S$. The main result of the present section is Theorem 1.11 where we prove that the ideal $\mathcal{A} A$ of $A$ is equal to the $A$-submodule $\left(g / y^{n}\right) K^{(n)}$ of the quotient field of A.

We identify an explicit generating set for $\mathcal{A}$ in Theorem 3.6. An explicit minimal generating set for the ideal $K^{(n)}$ may be found in Theorem 3.2.

Assume $n \geq 2$. Let $\varphi^{\prime}$ denote the restriction of $\varphi$ to $R(-d-1)^{m-2}$. We call $\varphi^{\prime}$ the linear part of $\varphi$ and we see that the image of $\varphi^{\prime}$ is the degree $d+1$ part of the first syzygy module of the $R$-module $I$. In other words,

$$
\varphi^{\prime}: R(-d-1)^{m-2} \rightarrow\left[\operatorname{syz}_{1}^{R}(I)\right]_{d+1}
$$

is an isomorphism. The row space of $\varphi^{\prime}, \operatorname{Row} \operatorname{Sp}\left(\varphi^{\prime}\right)$, is the $R$-module generated by the rows of $\varphi^{\prime}$. Observe that the minimal number of generators of the $R$ module $\operatorname{RowSp}\left(\varphi^{\prime}\right)$, denoted $\mu\left(\operatorname{RowSp}\left(\varphi^{\prime}\right)\right)$, depends only on $I$ and not on the set of generators $\left\{\delta_{i}\right\}$ for $I$ or the presenting matrix $\varphi$ of $I$. 
row

Definition 1.2. Given the resolution (1.1), with $n \geq 2$, let $\rho(I)$ be the parameter

$$
\rho=\mu\left(\operatorname{RowSp}\left(\varphi^{\prime}\right)\right)-m+2 .
$$

The hypothesis that $I$ has height two ensures that $m-1 \leq \mu\left(\operatorname{Row} \operatorname{Sp}\left(\varphi^{\prime}\right)\right)$; and therefore,

$$
1 \leq \rho \leq 2
$$

We have introduced the parameter $\rho$ at the present time for expository reasons; however, ultimately, $\rho$ plays a significant role in our study. For example, the value $\rho$ determines whether the fiber ring of $I$ is Cohen-Macaulay (see Theorem 4.2). It also determines many analytic properties about the powers of the ideal $I$, see sections 4 and 6 .

Start with some minimal resolution for $I$ :

$$
0 \rightarrow F_{1,1} \oplus F_{1,2} \rightarrow F_{0} \rightarrow I
$$

with $F_{0} \cong R(-d)^{m}, F_{1,1} \cong R(-d-1)^{m-2}$ and $F_{1,2} \cong R(-d-n)$. We prove in Proposition 2.1 that there exists a partition $\sigma$ of $m-2$ into $\rho$ pieces and there exist bases for $F_{0}$ and $F_{1,1}$ such that the linear part of $\varphi$ is equal to the $m \times(m-2)$ matrix

phi'

$$
\varphi^{\prime}= \begin{cases}{\left[\begin{array}{cc}
D_{\sigma_{1}} & 0 \\
0 & D_{\sigma_{2}}
\end{array}\right],} & \text { if } \rho=2, \\
{\left[\begin{array}{c}
D_{\sigma_{1}} \\
0
\end{array}\right],} & \text { if } \rho=1\end{cases}
$$

where $D_{a}$ is the $(a+1) \times a$ matrix

$$
D_{a}=\left[\begin{array}{cccc}
x & 0 & 0 & 0 \\
-y & x & 0 & 0 \\
0 & -y & \ddots & 0 \\
0 & 0 & \ddots & x \\
0 & 0 & 0 & -y
\end{array}\right]
$$

and

$$
\begin{cases}\boldsymbol{\sigma}=\left(\sigma_{1}, \sigma_{2}\right), \text { with } \sigma_{1} \geq \sigma_{2} \geq 1, \text { and } \sigma_{1}+\sigma_{2}=m-2, & \text { if } \rho=2, \text { or } \\ \boldsymbol{\sigma}=\sigma_{1}=m-2, & \text { if } \rho=1 .\end{cases}
$$

We give the variables $T_{j}$ of $S$ alternate names. Let

$$
\begin{cases}T_{1, j}=T_{j}, & \text { if } 1 \leq j \leq \sigma_{1}+1, \text { and } \\ T_{2, j}=T_{\sigma_{1}+1+j}, & \text { if } \rho=2 \text { and } 1 \leq j \leq \sigma_{2}+1\end{cases}
$$


CN Definition 1.5. Adopt the data of (1.1) with $\varphi=\left[\begin{array}{ll}\varphi^{\prime} & \varphi^{\prime \prime}\end{array}\right]$, where $\varphi^{\prime}$ is given in (1.3) and $\phi^{\prime \prime}$ is an $m \times 1$ matrix of homogeneous forms of degree $n$. Let $\psi$ be the $2 \times(m-1)$ matrix

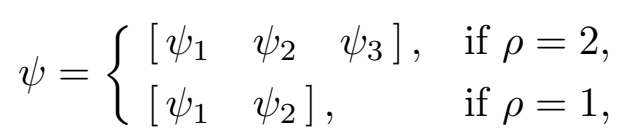

where each $\psi_{i}$ is a generic scroll matrix:

psi

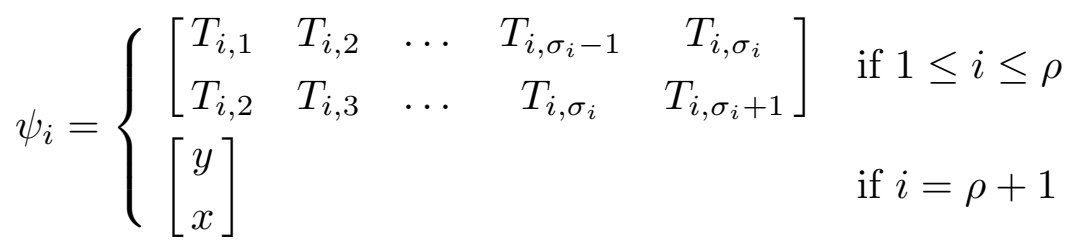

Let $H=I_{2}(\psi), A=S / H, \boldsymbol{T}$ be the matrix $\left[\begin{array}{lll}T_{1} & \ldots & T_{m}\end{array}\right], g \in S$ be the product $\boldsymbol{T} \varphi^{\prime \prime}, K$ be the ideal of $A$ which is generated by the entries in the top row of $\psi$, and $Q$ be the quotient field of $A$. The ideal $K$ is a prime ideal of $A$; let $K^{(n)}$ be the $n^{\text {th }}$ symbolic power of $K$.

It is convenient to think of the ring $S$ as bi-graded.

bi-

The variables $\left\{T_{i}\right\}$ have degree $(0,1)$.

The variables $\{x, y\}$ have degree $(1,0)$.

The last column of $\boldsymbol{T} \varphi$ has the form

$$
g=\sum_{i=0}^{n} c_{i} x^{n-i} y^{i} \in S
$$

where $c_{0}, \ldots, c_{n}$ are homogeneous elements of $S$ of degree $(0,1)$ and $g$ is a homogeneous polynomial of degree $(n, 1)$. The generators of $\mathcal{A}$ which are not in $\mathrm{H}$ are all described in terms of the polynomials $c_{0}, \ldots, c_{n}$; see Definition 3.5.

Remark. Let $V \subseteq \mathbb{P}^{m+1}$ be the variety defined by $I_{2}(\psi)$. We observe that when $\rho=1$, then the defining equations of $V$ do not involve the variable $T_{m}$. In other words, in this case, $V$ is the cone of a variety which lives in $\mathbb{P}^{m}$.

O5.1 Observation 1.9. The ideal $H$ and the polynomial $g$ are contained in $\mathcal{A}$.

Proof. The symmetric algebra $\operatorname{Sym}(I)$ is equal to

$$
\frac{S}{I_{1}(\boldsymbol{T} \varphi)}
$$


and the homomorphism $\Phi: S \rightarrow \mathcal{R}(I)$ factors through the natural quotient map $S \rightarrow \operatorname{Sym}(I) ;$ so,

$$
I_{1}(T \varphi) \subseteq \mathcal{A} .
$$

In particular, $g=\boldsymbol{T} \varphi^{\prime \prime}$ is in $\mathcal{A}$. Write $\psi=\left[\begin{array}{ll}\psi^{\prime} & \psi^{\prime \prime}\end{array}\right]$, where $\psi^{\prime}$ is first $m-2$ columns of $\psi$ and $\psi^{\prime \prime}$ is the final column of $\psi$. Observe that the product $\boldsymbol{T} \varphi^{\prime}$ is also equal to

here

$$
\left[\begin{array}{ll}
x & -y
\end{array}\right] \psi^{\prime} .
$$

Each entry of the matrix (1.10) is equal to a $2 \times 2$ minor of $\psi$ which involves the last column. Let $\delta$ be a $2 \times 2$ minor of $\psi$ which does not involve the last column of $\psi$. Cramer's rule, applied to (1.10), shows that $(x, y) \delta \subseteq \mathcal{A}$; but the ideal $\mathcal{A}$ is prime and $\mathcal{A} \cap R=\{0\}$; so $\delta$ is also in $\mathcal{A}$.

main1 Theorem 1.11. Retain the data of Definition 1.5. The A-submodule $\left(g / y^{n}\right) K^{(n)}$ of $Q$ is contained in $A$ and is equal to $\mathcal{A} A$.

Proof. If $T_{i} \in K$, then $T_{i} x=T_{i+1} y$ in $A$. It follows that $(x, y) K \subseteq(y) A$ and $(x, y)^{n} K^{n} \subseteq\left(y^{n}\right) A$. The divizorialization of the ideal $K^{n}$ in $A$ is $K^{(n)}$; hence, $\left(y^{n}\right): K^{n}=\left(y^{n}\right): K^{(n)}$ and

$$
g \in(x, y)^{n} \subseteq\left(y^{n}\right): K^{n}=\left(y^{n}\right): K^{(n)} .
$$

We conclude that the $A$-submodule $\left(g / y^{n}\right) K^{(n)}$ of $Q$ is contained in $A$. Let $L$ be the pre-image in $S$ of the ideal $\left(g / y^{n}\right) K^{(n)}$ of $A=S / H$. We see that

$$
y^{n} L \subseteq(g)+H \subseteq \mathcal{A} .
$$

The ideal $\mathcal{A}$ is prime and $y \notin \mathcal{A}$. Thus,

$$
H \subseteq L \subseteq \mathcal{A}
$$

are ideals of $S$ with $H$ and $\mathcal{A}$ are prime. Furthermore, the height of $H$ is $m-2$; the dimension of $S$ is $m+2$; and the dimension of the Rees ring $R[I t]$ is 3 . It follows that $\mathcal{A}$ has height $m-1$. The proof that $L=\mathcal{A}$ follows quickly from the following three facts. (This style of reasoning was suggested to us by [16].)

$6.17 \quad(1.12) g \in L \backslash H$,

$6.19 \quad(1.13) L$ is unmixed, and

$6.18 \quad(1.14) L \nsubseteq(H, x, y)$.

Indeed, (1.12) ensures that $L$ has height $m-1,(1.13)$ ensures that every associated prime of the $S$-module $S / L$ has height $m-1$. The ideal $(H, x, y)$ has height 
$m-1$ but is not an associated prime of $S / L$ by (1.14); hence, there is an element $w \in(x, y) k[x, y]$ which is regular on $S / L$. It follows that $I_{w}=R_{w}, \operatorname{Sym}(I)_{w}$ and $R[I t]_{w}$ are both polynomial rings in one variable over $R_{w}$, and the natural map $\operatorname{Sym}(I)_{w} \rightarrow R[I t]_{w}$ is an isomorphism. We have $I_{1}(\boldsymbol{T} \varphi)_{w} \subseteq L_{w} \subseteq \mathcal{A}_{w}$ and the the outer ideals are prime of the same height. We conclude that $L_{w}=\mathcal{A}_{w}$; and therefore, $L=\mathcal{A}$.

We establish the three facts, beginning with (1.12). The polynomial $g$ of degree $(n, 1)$ may be found in (1.8). Order the variables of $S$ by $T_{1}>\cdots>T_{m}>y>x$ and order the monomials of $S$ by the reverse lexicographic ordering. We know that the $\left(\begin{array}{c}m-1 \\ 2\end{array}\right)$ two by two minors of $\psi$ form a Gröbner basis for $H$ and we know that the initial ideal of $H$ is generated by

$$
\begin{cases} & \left\{T_{i, j} T_{i, k} \mid 1 \leq i \leq k \text { and } 2 \leq j \leq k \leq \sigma_{i}\right\} \\ \cup\left\{T_{1, i} T_{2, j} \mid 2 \leq i \leq \sigma_{1}+1 \text { and } 1 \leq j \leq \sigma_{2}\right\} & \\ \cup\left\{T_{i, j} y \mid 1 \leq i \leq 2 \text { and } 2 \leq j \leq \sigma_{i}+1\right\}, & \text { if } \rho=2, \text { and } \\ \left\{T_{1, j} T_{1, k} \mid 2 \leq j \leq k \leq \sigma_{1}\right\} \cup\left\{T_{1, j} y \mid 2 \leq j \leq \sigma_{1}+1\right\}, & \text { if } \rho=1 .\end{cases}
$$

We identify an element $h$ of $H$ for which the initial term of $g-h$ is either $T_{1} y^{n}$ or $T_{\sigma_{1}+2} y^{n}$. In either case, the initial term of $g-h$ is not in the initial ideal of $H$ (recall from(1.4) that some of the variables of $S$ have two names); therefore, $g-h \notin H$ and $g \notin H$. The linear form $c_{n}$ is $\sum_{i=1}^{m} \alpha_{i} T_{i}$ for some $\alpha_{i} \in k$. Recall the matrix $\varphi$ from (1.5). The hypothesis that height $I_{m-1}(\varphi)=2$ forces $\alpha_{1}$ or $\alpha_{\sigma_{1}+2}$ to be non-zero. Indeed, if $\alpha_{1}$ and $\alpha_{\sigma_{1}+2}$ are both zero, then the $(1, m-1)$ and $\left(\sigma_{1}+2, m-1\right)$ entries of $\varphi$ are both elements of the ideal $(x)$, the rank of the matrix $\left.\varphi\right|_{x=0}$ is $m-2$, and $I_{m-1}(\varphi) \subseteq(x)$. If $\alpha_{1} \neq 0$, then take $h=0$. The initial term of $g$ is $\alpha_{1} T_{1} y^{n}$ and the proof of (1.12) is complete in this case. If $\alpha_{1}=0$, then $\alpha_{\sigma_{1}+2} \neq 0$ and we take

$$
h=\sum_{i=2}^{\sigma_{1}+1} y^{n-1} \alpha_{i}\left(y T_{i}-x T_{i-1}\right) .
$$

The initial term of $g-h$ is $\alpha_{\sigma_{1}+2} T_{\sigma_{1}+2} y^{n}$ and the proof of (1.12) is complete in all cases.

Now we prove (1.13). The ideal $K$ is a height one prime ideal of the normal domain $A$ and therefore the symbolic power $K^{(n)}$ is a divisorial ideal of $A$. To show that the ideal $L$ of $S$ is unmixed, it suffices to prove that the height one ideal $L A$ of $A$ is isomorphic, as an $A$-module, to the divisorial ideal $K^{(n)}$ of $A$. We saw in (1.12) that $g \notin H$. Thus, $g / y^{n}$ is a non-zero element of the fraction field $Q$ of $A$ and

GL $\quad(1.15)$

multiplication $g / y^{n}: K^{(n)} \rightarrow L A$ is an $A$-module isomorphism. 
Finally, we prove (1.14). Recall that $Q$ is the fraction field of $A$. We know from (1.15) that the $A$-submodules $L A$ and $\frac{g}{y^{n}} K^{(n)}$ of $Q$ are equal. We show (1.14) by showing that $\frac{g}{y^{n}} K^{(n)} \nsubseteq(x, y) A$. We compute

$$
(x, y) A:{ }_{Q} K^{(n)} .
$$

Our notation means that $(x, y) A$ and $K^{(n)}$ are $A$-submodules of $Q$ and : is calculated in $Q$; that is,

$$
(x, y) A:_{Q} K^{(n)}=\left\{\alpha \in Q \mid \alpha K^{(n)} \subseteq(x, y) A\right\} .
$$

Notice first that $(x, y)=(y):{ }_{Q} K$. The element $y$ is in $K ;$ so, $(y):{ }_{Q} K$ is an ideal of $A$ and the assertion $(x, y)=(y):{ }_{Q} K$ is a statement about geometric linkage. The ideal $(x, y)^{n+1} A$ is divisorial; so we have

$$
\begin{aligned}
& (x, y) A:_{Q} K^{(n)}=\left((y):_{Q} K\right):{ }_{Q} K^{(n)}=(y):_{Q} K K^{(n)}=y^{-n}\left[\left(y^{n+1}\right):_{Q} K K^{(n)}\right] \\
& =y^{-n}\left[\left(y^{n+1}\right):{ }_{Q} K^{n+1}\right]=y^{-n}(y, x)^{n+1} A .
\end{aligned}
$$

If the polynomial $g$, of degree $(n, 1)$, were in $(x, y)^{n+1} S+H$, then degree considerations show that $g$ would be in $H$, and this possibility was ruled out in (1.12). Thus, $g / y^{n} \notin \frac{1}{y^{n}}(y, x)^{n+1}=(x, y) A:{ }_{Q} K^{(n)}$ and the proof is complete.

\section{Matrices with linear entries.}

Let $\varphi$ be the matrix of (1.1). In Proposition 2.1 we prove that there exist row and column operations on $\varphi$ which transform the linear part of $\varphi$ into a matrix of the form described in (1.3). Fix the polynomial ring $R=k[x, y]$ over the field $k$. For each non-negative integer $a$, let $D(a)$ be the $(a+1) \times a$ matrix with

$$
D(a)_{i, j}= \begin{cases}x, & \text { if } i=j \text { and } 1 \leq j \leq a, \\ y, & \text { if } i=j+1 \text { and } 1 \leq j \leq a, \text { and } \\ 0 & \text { otherwise. }\end{cases}
$$

We see that $D(0)$ is invisible,

$$
D(1)=\left[\begin{array}{l}
x \\
y
\end{array}\right], \text { and } D(2)=\left[\begin{array}{ll}
x & 0 \\
y & x \\
0 & y
\end{array}\right] .
$$

The matrix $D_{a}$ of section 1 is the same as the matrix $D(a)$ of the present section, with $y$ replaced by $-y$. 
P28.1 Proposition 2.1. Let $M$ be an $m \times(m-2)$ matrix whose entries are homogeneous linear forms from $R$. Suppose that there exists a column vector $\varphi^{\prime \prime}$ in $R^{m}$ of homogeneous forms of the same degree, such that the ideal of maximal minors of $\left[\begin{array}{ll}M & \varphi^{\prime \prime}\end{array}\right]$ is an ideal of height two in $R$. Then there exist matrices $A \in \mathrm{GL}_{m}(k)$ and $B \in \mathrm{GL}_{m-2}(k)$ and non-negative integers $b \leq a$, with $a+b=m-2$, such that

dab

$$
A M B=\left[\begin{array}{cc}
D(a) & 0 \\
0 & D(b)
\end{array}\right] .
$$

Remark. If $b=0$ and $a=m-2$, then the matrix on the right side of (2.2) is

$$
\left[\begin{array}{c}
D(m-2) \\
0
\end{array}\right]
$$

where 0 represents a $1 \times m-2$ matrix of zeros. Observe that $\mu(\operatorname{RowSp}(M))=m-1$ and the parameter $\rho$ of (1.2) is 1 . In the language of (1.3), this is the situation in which the partition $\boldsymbol{\sigma}$ of $m-2$ consists of 1 piece $\boldsymbol{\sigma}=(m-2)$.

Proof. We first translate the hypothesis that $I_{m-1}(\varphi)$ has height two (for $\varphi$ equal to $\left[\begin{array}{ll}M & \varphi^{\prime \prime}\end{array}\right]$ ) into a statement strictly about $M$. The ideal $I_{m-1}(\varphi)$ is not affected by row and column operations on $\varphi$; so, the ideal of maximal minors of $\left[\begin{array}{lll}A M B & A \varphi^{\prime \prime}\end{array}\right]$ has height two for all $A \in \mathrm{GL}_{m}(k)$ and $B \in \mathrm{GL}_{m-2}(k)$. In particular, each column of $A M B$ generates an ideal of height two. In fact, however, according to Lemma 2.7 , even more is true:

cond

$$
\text { If } A \in \mathrm{GL}_{m}(k) \text { and } B \in \mathrm{GL}_{m-2}(k) \text {, then } A M B \text { does not contain }
$$

$$
\begin{aligned}
& \text { a } p \times q \text { submatrix of zeros for any pair of positive integers }(p, q) \\
& \text { with } p+q=m \text {. }
\end{aligned}
$$

The proof of the present result is by induction on $m$. The assertion is obvious when $m=3$. Henceforth, $4 \leq m$. Let $\bar{M}$ be the image of $M$ in the $\operatorname{ring} R /(y)$. We see that $\bar{M}=x M^{\prime}$ for some $m \times(m-2)$ matrix $M^{\prime}$ with entries in $k$. The matrix $M^{\prime}$ must have rank $m-2$; otherwise, there exist invertible matrices $A$ and $B$ so that the entries of one column of $A M B$ all are in the ideal $(y)$. It follows that there exist invertible matrices $A$ and $B$ with

$$
A M^{\prime} B=\left[\begin{array}{c}
I_{m-2} \\
0_{2 \times(m-2)}
\end{array}\right]
$$

and therefore every entry in the bottom two rows of $A M B$ is in the ideal $(y)$. Some entry of the bottom two rows of $A M B$ is not zero by (2.3). Thus, further row and column operations yield a matrix of the form

$$
\left[\begin{array}{cc}
M_{1} & M_{2} \\
0 & y
\end{array}\right]
$$


The $(m-1) \times(m-3)$ matrix $M_{1}$ satisfies $(2.3)$ because if there exist $A_{1} \in \mathrm{GL}_{m-1}(k)$ and $B_{1} \in \mathrm{GL}_{m-3}(k)$ so that $A_{1} M_{1} B_{1}$ contains an $p_{1} \times q_{1}$ zero submatrix, then there exist invertible matrices $A$ and $B$ so that $A M B$ contains an $\left(p_{1}+1\right) \times q_{1}$ zero submatrix.

By induction $M$ may be transformed into

form

$$
\left[\begin{array}{ccc}
D(a) & 0 & C_{1} \\
0 & D(b) & C_{2} \\
0 & 0 & y
\end{array}\right]
$$

for two non-negative integers $b \leq a$ with $a+b=m-3$, where $C_{1}$ and $C_{2}$ are column vectors. Use column operations to remove all $x$ 's from $C_{1}$ and $C_{2}$, except possibly in the bottom row. Use row operations to remove all $y$ 's from $C_{1}$ and $C_{2}$. Thus, $M$ may be transformed into a matrix of the form (2.4) with

$$
C_{i}=\left[\begin{array}{c}
0 \\
\vdots \\
0 \\
c_{i} x
\end{array}\right]
$$

for some $c_{i} \in k$. At least one of the constants $c_{1}$ or $c_{2}$ must be non-zero. If $c_{1}$ is not zero, then pre-multiply and post-multiply by

$$
A=\left[\begin{array}{ccc}
c_{1}^{-1} I & 0 & 0 \\
0 & I & 0 \\
0 & 0 & 1
\end{array}\right] \text { and } B=\left[\begin{array}{ccc}
c_{1} I & 0 & 0 \\
0 & I & 0 \\
0 & 0 & 1
\end{array}\right]
$$

respectively, to transform $c_{1}$ into 1 . The constant $c_{2}$ may be treated in a similar manner. Thus, $M$ may be transformed into a matrix of form (2.4) with (2.5) and one of three cases:

$$
\begin{cases}c_{1}=1, c_{2}=0 & \text { case } 1 \\ c_{1}=0, c_{2}=1 & \text { case } 2 \\ c_{1}=c_{2}=1 & \text { case } 3\end{cases}
$$

The third case may be transformed into the second case using

$$
A=\left[\begin{array}{cccc}
I_{a-b} & 0 & 0 & 0 \\
0 & I_{b+1} & -I_{b+1} & 0 \\
0 & 0 & I_{b+1} & 0 \\
0 & 0 & 0 & 1
\end{array}\right] \text { and } B=\left[\begin{array}{cccc}
I_{a-b} & 0 & 0 & 0 \\
0 & I_{b} & I_{b} & 0 \\
0 & 0 & I_{b} & 0 \\
0 & 0 & 0 & 1
\end{array}\right]
$$


In the second case, (2.4) is readily seen to be

$$
\left[\begin{array}{cc}
D(a) & 0 \\
0 & D(b+1)
\end{array}\right]
$$

and in the first case, one may rearrange the rows and columns of (2.4) to obtain

$$
\left[\begin{array}{cc}
D(a+1) & 0 \\
0 & D(b)
\end{array}\right]
$$

Finally, we notice that if $b+1>a$, then one may rearrange the rows and columns of (2.6) to obtain

$$
\left[\begin{array}{cc}
D(b+1) & 0 \\
0 & D(a)
\end{array}\right]
$$

'a+b Lemma 2.7. Let $\varphi$ be an $m \times(m-1)$ matrix with entries from some commutative ring. Suppose that there are positive integers $p$ and $q$ with $p+q=m$ and

$$
\varphi=\left[\begin{array}{ll}
Z & S \\
T & U
\end{array}\right]
$$

where $Z$ is an $p \times q$ matrix of zeros and $S, T$, and $U$ are matrices. Then the ideal $I_{m-1}(\varphi)$ is contained in the principal ideal $(\operatorname{det} T)$.

Proof. It suffices to establish this result in the generic situation where the ring is a polynomial ring over the integers and each entry of $\varphi$ is an indeterminate. The matrices $S, T$, and $U$, have shape $p \times(p-1), q \times q$, and $q \times(p-1)$ respectively. Let $\varphi_{i}$ (respectively $S_{i}$ ) represent the determinant of $\varphi$ (resp. $S$ ) with row $i$ deleted. If $p<i$, then the rows $\left[\begin{array}{ll}Z & S\end{array}\right]$ are linearly dependent and $\varphi_{i}=0$. If $i \leq p$, then $\varphi_{i}= \pm S_{i} \operatorname{det} T$.

7-28 Observation 2.8. One can arrange the data of Definition 1.5 so that the term $y^{n} T_{1}$ appears in $g$ with a non-zero coefficient.

Proof. It suffices to show that one may modify $\varphi$ in order to have $\varphi_{1}^{\prime \prime} \notin(x)$, keeping $\varphi^{\prime}$ unchanged.

Proposition 2.1 shows how to apply row and column operations to the matrix $\varphi=\left[\begin{array}{ll}M & \varphi^{\prime \prime}\end{array}\right]$ in order to to produce the matrix $\left[\begin{array}{lll}A M B & A \varphi^{\prime \prime}\end{array}\right]$, where $A M B$ has the form of (2.2). It is possible to apply more row and column operations in order to guarantee that the top element of $A \varphi^{\prime \prime}$ is not in the ideal $(x)$. Indeed, it is shown in the proof of $(1.12)$ that it is not possible for $\left(A \varphi^{\prime \prime}\right)_{1}$ and $\left(A \varphi^{\prime \prime}\right)_{a+2}$ to both be 
in $(x)$. If $\left(A \varphi^{\prime \prime}\right)_{1} \in(x)$, then add a copy of row $a+2$ to row 1 . This row operation may transform

$$
\left[\begin{array}{cc}
D(a) & 0 \\
0 & D(b)
\end{array}\right] \text { into }\left[\begin{array}{cc}
D(a) & x E_{1,1} \\
0 & D(b)
\end{array}\right]
$$

where $E_{i, j}$ is the $(a+1) \times b$ elementary matrix with 1 in position $(i, j)$ and 0 elsewhere. One can remove the unwanted $x$ using row and column operations which do not involve row one. The first step, subtract column 1 from column $a+1$ and add row $a+3$ to row 2 , transforms

$$
\left[\begin{array}{cc}
D(a) & x E_{1,1} \\
0 & D(b)
\end{array}\right] \text { into }\left[\begin{array}{cc}
D(a) & x E_{2,2} \\
0 & D(b)
\end{array}\right]
$$

without harming the top entry in the augmented column. One repeats this trick until the $x$ slides off the edge.

C2.4 Corollary 2.9. If $I$ is a height two ideal in $R=k[x, y]$, then the resolution of $I$ is given in (1.1) if and only if there exists non-negative integers $a$ and $b$, with $a+b=m-2$, and homogeneous forms $F_{1}$ and $F_{2}$ in $R$, with $\operatorname{deg} F_{1}=n+a$ and $\operatorname{deg} F_{2}=n+b$ such that

$$
I=(x, y)^{b} F_{1}+(x, y)^{a} F_{2} .
$$

Proof. Start with the data $a, b, F_{1}$, and $F_{2}$. Write

$$
F_{1}=\sum_{i=0}^{a} \alpha_{i} x^{a-i} y^{i} \quad \text { and } \quad F_{2}=\sum_{i=0}^{b} \beta_{i} x^{b-i} y^{i},
$$

for homogenous forms $\alpha_{i}$ and $\beta_{i}$ of degree $n$. Let

$$
\boldsymbol{\alpha}=\left[\begin{array}{c}
\alpha_{a} \\
\vdots \\
\alpha_{0}
\end{array}\right] \quad \text { and } \quad \boldsymbol{\beta}=\left[\begin{array}{c}
\beta_{b} \\
\vdots \\
\beta_{0}
\end{array}\right] \text {. }
$$

Observe that

$$
\operatorname{det}\left[\begin{array}{ll}
D_{a} & \boldsymbol{\alpha}
\end{array}\right]=F_{1} \quad \operatorname{det}\left[\begin{array}{ll}
D_{b} & \boldsymbol{\beta}
\end{array}\right]=F_{2},
$$

and the ideal generated by the maximal order minors of the matrix

$$
\left[\begin{array}{ccc}
D_{a} & 0 & \boldsymbol{\alpha} \\
0 & D_{b} & \boldsymbol{\beta}
\end{array}\right]
$$

is equal to $I$.

The converse is established in Proposition 2.1. 
E2.5 Example 2.10. If $F_{1}=y^{n+a}$ and $F_{2}=x^{n+b}$, then $I$ is the monomial ideal

$$
\left(y^{d}, x y^{d-1}, \ldots, x^{b} y^{d-b}\right)+\left(x^{d-a} y^{a}, \ldots, x^{d-1} y, x^{d}\right) .
$$

The following proof was prompted to us by a question of Craig Huneke.

C2.6 Corollary 2.11. Adopt the notation of Corollary 2.9 with $b \leq a$, and write $d=$ $n+a+b$. For every $s \geq 1$ one has

$$
\operatorname{reg} I^{s}=\max \{s d, s d-(s-1) b+n-1\} .
$$

Proof. Write $\mathfrak{m}=(x, y)$. Notice that the regularity of a homogeneous $\mathfrak{m}$-primary ideal is the smallest power of $\mathfrak{m}$ contained in it. Notice that $I^{s}$ is generated by forms of degree $s d$ and

$$
I^{s}=\sum_{i=0}^{s} \mathfrak{m}^{s d-\operatorname{deg}\left(F_{1}^{i} F_{2}^{s-i}\right)} F_{1}^{i} F_{2}^{s-i} \subset\left(F_{1}, F_{2}\right)^{s} \cap \mathfrak{m}^{s d} .
$$

Hence $\mathfrak{m}^{t} \subset I^{s}$ if and only if $\mathfrak{m}^{t} \subset\left(F_{1}, F_{2}\right)^{s}$ and $t \geq s d$. In other words,

$$
\operatorname{reg} I^{s}=\max \left\{s d, \operatorname{reg}\left(F_{1}, F_{2}\right)^{s}\right\} .
$$

Finally, $F_{1}, F_{2}$ are a regular sequence of forms of degrees $n+b \leq n+a$. Hence $\left(F_{1}, F_{2}\right)^{s}$ is presented by the $s+1$ by $s$ matrix

$$
\left[\begin{array}{cccccc}
F_{2} & & & & \\
-F_{1} & F_{2} & & & \\
& -F_{1} & \cdot & & \\
& & \cdot & \cdot & & \\
& & & \cdot & \cdot & \\
& & & & \cdot & F_{2} \\
& & & & -F_{1}
\end{array}\right]
$$

From this minimal homogeneous resolution one sees that

$$
\operatorname{reg}\left(F_{1}, F_{2}\right)^{s}=s(n+a)+n+b-1=s d-(s-1) b+n-1 .
$$

It is shown in $[10,21,28]$ that the regularity of $I^{s}$ is a linear function of $s$ for all $s \gg 0$. Indeed, in our notation, the aforementioned papers guarantee that $\operatorname{reg}\left(I^{s}\right)=s d+e$ for some non-negative integer $e$. From Corollary 2.11, we read the exact value of $e$ and the least value of $s$ for which the above equation holds. The answers depend on the value of $\rho$. In Section 6 we resolve each power $I^{s}$; thereby confirming the present calculations, see especially Corollary 6.8 . 
C2.7 Corollary 2.12. Let $I$ be the ideal of Definition 1.5 and $s$ be a positive integer.

(1) If $\rho=1$, then $\operatorname{reg} I^{s}=s d+n-1$ for all $s \geq 1$.

(2) If $\rho=2$, then $\operatorname{reg} I^{s}=s d$ if and only if $\frac{n-1}{\sigma_{2}}+1 \leq s$.

Proof. If $\rho=1$, then the parameter $b$ of Corollary 2.11 is equal to zero and $\max \{s d, s d-(s-1) b+n-1\}$ is equal to $s d+n-1$ for all $s \geq 1$. If $\rho=2$, then the parameter $b$ of Corollary 2.11 is equal to $\sigma_{2}$ and reg $I^{s}=s d$ if and only if $s d \geq s d-(s-1) \sigma_{2}+n-1$.

\section{Explicit generators for the defining ideal of the Rees algebra.}

The main result of this section is Theorem 3.6 where we identify an explicit generating set for the defining ideal $\mathcal{A}$ of the Rees algebra $\mathcal{R}(I)$. Adopt the data of Definition 1.5 with

$$
\ell=\rho+1, \quad \sigma_{\ell}=1, \quad y=T_{\ell, 1}, \quad \text { and } \quad x=T_{\ell, 2} .
$$

In this notation, the matrix $\psi_{\ell}$ of (1.6) is

$$
\psi_{\ell}=\left[\begin{array}{l}
T_{\ell, 1} \\
T_{\ell, 2}
\end{array}\right] .
$$

Accoding to Theorem 1.11, we need to identify generators for the ideal $L$ in $S$ with $g K^{(n)}=y^{n} L A$. The following minimal generating set for $K^{(n)}$ is calculated in [22].

Kupn Theorem 3.2. A $k$-tuple $\boldsymbol{a}=\left(a_{1}, \ldots, a_{k}\right)$ of non-negative integers is eligible if $0 \leq k \leq \rho$ and $\sum_{u=1}^{k} a_{u} \sigma_{u}<n$. If $\boldsymbol{a}$ is an eligible $k$-tuple, then $f(\boldsymbol{a})$ and $r(\boldsymbol{a})$ are defined by:

$$
\sum_{u=1}^{k} a_{u} \sigma_{u}+f(\boldsymbol{a}) \sigma_{k+1}<n \leq \sum_{u=1}^{k} a_{u} \sigma_{u}+(f(\boldsymbol{a})+1) \sigma_{k+1}
$$

and

$$
r(\boldsymbol{a})=\sum_{u=1}^{k} a_{u} \sigma_{u}+(f(\boldsymbol{a})+1) \sigma_{k+1}-n+1 .
$$

The ideal $K^{(n)}$ of $A$ is equal to

$$
K^{(n)}=\left(\left\{T^{\boldsymbol{a}} T_{k+1,1}^{f(\boldsymbol{a})} T_{k+1, j} \mid \boldsymbol{a} \text { is an eligible } k \text {-tuple and } 1 \leq j \leq r(\boldsymbol{a})\right\}\right) A,
$$

where $T^{\boldsymbol{a}}=\prod_{u=1}^{k} T_{u, 1}^{a_{u}}$.

Remark. The empty tuple, $\emptyset$, is always eligible, and we have

$$
f(\emptyset)=\left\lceil\frac{n}{\sigma_{1}}\right\rceil-1, \quad r(\emptyset)=\sigma_{1}\left\lceil\frac{n}{\sigma_{1}}\right\rceil-n+1, \quad \text { and } \quad T^{\emptyset}=1 .
$$


-1D6.1 Definition 3.3. Recall the polynomials $c_{0}, \ldots, c_{n}$ of (1.8).

(a) For integers $a$ and $b$ with $a+b \leq n$ and $0 \leq a$, define the polynomial $\Delta_{a, b}$ to be

$$
\begin{cases}\sum_{k=0}^{b} c_{a+k} x^{b-k} y^{k}=c_{a} x^{b}+c_{a+1} x^{b-1} y+\cdots+c_{a+b} y^{b}, & \text { if } 0 \leq b, \\ 0, & \text { if } b<0 .\end{cases}
$$

In particular $g=\Delta_{0, n}$. Furthermore, $\Delta_{a, b}$ is a homogeneous element of $S$ of degree $(b, 1)$.

(b) If $0 \leq a \leq n$, then write $\Delta_{a}$ to mean $\Delta_{a, n-a}$. So

$$
\Delta_{a}=c_{a} x^{n-a}+c_{a+1} x^{n-a-1} y+\cdots+c_{n} y^{n-a},
$$

and $\Delta_{a}$ is a homogeneous element of $S$ of degree $(n-a, 1)$.

(c) For each 4-tuple of non-negative indices $(i, a, b, \gamma)$ with

$$
1 \leq i \leq \rho, \quad b+1 \leq \gamma \leq \sigma_{i}+1, \quad \text { and } \quad a+b \leq n,
$$

define $\pi_{i, a, b, \gamma} \in S$ of degree $(0,2)$ to be

$$
\pi_{i, a, b, \gamma}=\sum_{k=0}^{b} c_{a+k} T_{i, \gamma-k}=c_{a} T_{i, \gamma}+c_{a+1} T_{i, \gamma-1}+\cdots+c_{a+b} T_{i, \gamma-b} .
$$

(d) If $1 \leq i \leq \rho$ and $0 \leq a \leq n-\sigma_{i}+1$, then let $\pi_{i, a}$ mean $\pi_{i, a, \sigma_{i}-1, \sigma_{i}+1}$; so $\pi_{i, a}$ is equal to

$$
\sum_{k=0}^{\sigma_{i}-1} c_{a+k} T_{i, \sigma_{i}+1-k}=c_{a} T_{i, \sigma_{i}+1}+c_{a+1} T_{i, \sigma_{i}}+\cdots+c_{a+\sigma_{i}-1} T_{i, 2} .
$$

(e) If $(i, s, j)$ are non-negative integers with $1 \leq i \leq \rho$ and $1 \leq j \leq \sigma_{i}+1-s$, then let $\pi_{i, s, j}^{\prime}$ mean $\pi_{i, n-s, s, s+j}$.

1R6.8 Remarks 3.4. (a) Reverse the order of summation in the polynomial $\pi_{i, s, j}^{\prime}$ to write

$$
\pi_{i, s, j}^{\prime}=\sum_{k=0}^{s} c_{n-k} T_{i, j+k}=c_{n-s} T_{i, j+s}+\cdots+c_{n-1} T_{i, j+1}+c_{n} T_{i, j} .
$$

(b) If the non-negative integers $a, b, \gamma$ satisfy $a+b \leq n$ and $1 \leq \gamma \leq b$, then

$$
\Delta_{a, b}=x^{b-\gamma+1} \Delta_{a, \gamma-1}+y^{\gamma} \Delta_{a+\gamma, b-\gamma} .
$$


The polynomial $\Delta_{a, b}$ of is homogeneous in $x$ and $y$ of degree $b$. This formula amounts to the statement that every term in $\Delta_{a, b}$ is divisible by either $y^{\gamma}$ or $x^{b-\gamma+1}$. At any rate, the left side is

$$
\begin{gathered}
\left(c_{a} x^{b}+\cdots+c_{a+\gamma-1} x^{b-\gamma+1} y^{\gamma-1}\right)+\left(c_{a+\gamma} x^{b-\gamma} y^{\gamma}+\cdots+c_{a+b} y^{b}\right) \\
=x^{b-\gamma+1}\left(c_{a} x^{\gamma-1}+\cdots+c_{a+\gamma-1} y^{\gamma-1}\right)+y^{\gamma}\left(c_{a+\gamma} x^{b-\gamma}+\cdots+c_{a+b} y^{b-\gamma}\right),
\end{gathered}
$$

which is the right side.

(c) If $N$ is negative, then the sum $\sum_{a+b=N}$ is zero; if $N$ is a non-negative integer then the sum $\sum_{a+b=N}$ is taken over all pairs of non-negative integers $(a, b)$, with $a+b=N$.

(d) We calculate in $S$. If $s_{1}$ and $s_{2}$ are elements of $S$, we write $s_{1} \equiv s_{2}$ to mean that $s_{1}-s_{2} \in H$.

1D5.3 Definition 3.5. For each pair $(\boldsymbol{a}, j)$, where $\boldsymbol{a}$ is an eligible tuple and $1 \leq j \leq r(\boldsymbol{a})$, we define a polynomial $G_{(\boldsymbol{a}, j)}$ in $S$.

(a) If $1 \leq j \leq r(\emptyset)$, then let

$$
G_{(\emptyset, j)}=f_{j}=T_{1, \sigma_{1}+1-r(\emptyset)+j} \sum_{p+q=f(\emptyset)-1} T_{1,1}^{p} T_{1, \sigma_{1}+1}^{q} \pi_{1, p \sigma_{1}}+T_{1,1}^{f(\emptyset)} \pi_{1, \sigma_{1}+1-r(\emptyset), j}^{\prime} .
$$

(b) If $\left(a_{1}\right)$ is an eligible 1-tuple, and $1 \leq j \leq r\left(a_{1}\right)$, then let

$$
G_{\left(\left(a_{1}\right), j\right)}=g_{a_{1}, j}=\left\{\begin{array}{l}
T_{2, j+\sigma_{2}+1-r\left(a_{1}\right)} T_{2, \sigma_{2}+1}^{f\left(a_{1}\right)} \sum_{p+q=a_{1}-1} T_{1,1}^{p} T_{1, \sigma_{1}+1}^{q} \pi_{1, p \sigma_{1}} \\
+T_{1,1}^{a_{1}} T_{2, j+\sigma_{2}+1-r\left(a_{1}\right)} \sum_{p+q=f\left(a_{1}\right)-1} T_{2,1}^{p} T_{2, \sigma_{2}+1}^{q} \pi_{2, a_{1} \sigma_{1}+p \sigma_{2}} \\
+T_{1,1}^{a_{1}} T_{2,1}^{f\left(a_{1}\right)} \pi_{2, \sigma_{2}+1-r\left(a_{1}\right), j}^{\prime} .
\end{array}\right.
$$

(c) If $\boldsymbol{a}=\left(a_{1}, a_{2}\right)$ is an eligible 2-tuple, then $r(\boldsymbol{a})=1$. Let

$$
G_{(\boldsymbol{a}, 1)}=h_{a_{1}, a_{2}}=\left\{\begin{array}{l}
x^{n-a_{1} \sigma_{1}-a_{2} \sigma_{2}} T_{2, \sigma_{2}+1}^{a_{2}} \sum_{p+q=a_{1}-1} T_{1,1}^{p} T_{1, \sigma_{1}+1}^{q} \pi_{1, p \sigma_{1}} \\
+x^{n-a_{1} \sigma_{1}-a_{2} \sigma_{2}} T_{1,1}^{a_{1}} \sum_{p+q=a_{2}-1} T_{2,1}^{p} T_{2, \sigma_{2}+1}^{q} \pi_{2, a_{1} \sigma_{1}+p \sigma_{2}} \\
+T_{1,1}^{a_{1}} T_{2,1}^{a_{2}} \Delta_{a_{1} \sigma_{1}+a_{2} \sigma_{2}} .
\end{array}\right.
$$

(d) The ideal $L$ of $S$ is equal to

$$
H+\left(\left\{G_{(\boldsymbol{a}, j)} \mid \boldsymbol{a} \text { is an eligible tuple and } 1 \leq j \leq r(\boldsymbol{a})\right\}\right) .
$$

We are now able to state the main result of this section. The ideal $\mathcal{A}$ which defines the Rees algebra $\mathcal{R}(I)$ was introduced in the first paragraph of section 1 . 
$\mathrm{A}=\mathrm{L} \quad$ Theorem 3.6. The ideals $\mathcal{A}$ and $L$ of the ring $S$ are equal.

Proof. In light of Theorem 1.11, we need only show that $L A$ is equal to the $A$ submodule of $\left(g / y^{n}\right) K^{(n)}$ of $Q$. This calculation is carried out in Lemma 3.10.g.

1R6.1 Remarks 3.7.

(a) Observe that

$$
\begin{array}{ll}
f_{j} & \text { is homogeneous of degree }(0, f(\emptyset)+2), \\
g_{a_{1}, j} & \text { is homogeneous of degree }\left(0, a_{1}+f\left(a_{1}\right)+2\right), \text { and } \\
h_{a_{1}, a_{2}} & \text { is homogeneous of degree }\left(f\left(a_{1}, a_{2}\right)+1, a_{1}+a_{2}+1\right) .
\end{array}
$$

(b) Let $0^{s}$ be the $s$-tuple $(0, \ldots, 0)$. Observe that $G_{\left(0^{\rho}, 1\right)}=g$. Indeed, if $\rho=2$, then $h_{0,0}=\Delta_{0} g$, and if $\rho=1$, then

$$
\begin{aligned}
g_{0,1} & =T_{2,2} \sum_{p+q=n-2} T_{2,1}^{p} T_{2,2}^{q} \pi_{2, p}+T_{2,1}^{n-1} \pi_{2, n-1,1,2} \\
& =x \sum_{p+q=n-2} y^{p} x^{q} c_{p}+y^{n-1}\left(c_{n-1} x+c_{n} y\right)=g .
\end{aligned}
$$

1t6.7 Observation 3.8. If $a, i$, and $j$ are integers with $0 \leq a, 1 \leq i \leq \rho, 1 \leq j$, and $j+a \leq \sigma_{i}+1$, then $x^{a} T_{i, j} \equiv y^{a} T_{i, j+a}$.

Proof. The ideal

$$
I_{2}\left[\begin{array}{cccccc}
T_{i, 1} & T_{i, 2} & \cdots & T_{i, \sigma_{i}-1} & T_{i, \sigma_{i}} & y \\
T_{i, 2} & T_{i, 3} & \cdots & T_{i, \sigma_{i}} & T_{i, \sigma_{i}+1} & x
\end{array}\right]
$$

is contained in $H$. A quick induction completes the proof.

112.22 Observation 3.9. Take $1 \leq i \leq \rho$.

(a) If $0 \leq a \leq n-\sigma_{i}+1$, then $T_{i, 1} x \Delta_{a, \sigma_{i}-1} \equiv y^{\sigma_{i}} \pi_{i, a}$.

(b) If $0 \leq s \leq n$ and $1 \leq j \leq \sigma_{i}-s+1$, then $T_{i, j} \Delta_{n-s} \equiv y^{s} \pi_{i, s, j}^{\prime}$.

Proof. Use Observation 3.8 to see the left side of (a) is

$$
\sum_{k=0}^{\sigma_{i}-1} c_{a+k}\left(x^{\sigma-k} T_{i, 1}\right) y^{k} \equiv \sum_{k=0}^{\sigma_{i}-1} c_{a+k}\left(y^{\sigma_{i}-k} T_{i, \sigma_{i}-k+1}\right) y^{k}=y^{\sigma_{i}} \sum_{k=0}^{\sigma_{i}-1} c_{a+k} T_{i, \sigma_{i}-k+1},
$$

and this is the right side of (a). In a similar manner, we see that the left side of (b) is

$$
\sum_{k=0}^{s} c_{n-s+k}\left(T_{j} x^{s-k}\right) y^{k} \equiv \sum_{k=0}^{s} c_{n-s+k} T_{i, j+s-k} y^{s-k} y^{k}=y^{s} \pi_{i, n-s, s, j+s}=y^{s} \pi_{i, s, j}^{\prime} .
$$




\section{L46.1 Lemma 3.10.}

(a) If $\left(a_{1}, 0\right)$ and $\left(a_{1}+1,0\right)$ are eligible tuples, then $T_{1,1} h_{a_{1}, 0} \equiv y^{\sigma_{1}} h_{a_{1}+1,0}$.

(b) If $\left(a_{1}, a_{2}\right)$ and $\left(a_{1}, a_{2}+1\right)$ are eligible tuples, then $T_{2,1} h_{a_{1}, a_{2}} \equiv y^{\sigma_{2}} h_{a_{1}, a_{2}+1}$.

(c) If $\left(a_{1}\right)$ is eligible, $\rho=2$, and $1 \leq j \leq r\left(a_{1}\right)$, then

$$
T_{2, j} h_{a_{1}, f\left(a_{1}\right)} \equiv y^{\sigma_{2}+1-r\left(a_{1}\right)} g_{a_{1}, j} .
$$

(d) If $\rho=2$ and $1 \leq j \leq r(\emptyset)$, then $T_{1, j} h_{f(\emptyset), 0} \equiv y^{\sigma_{1}+1-r(\emptyset)} f_{j}$.

(e) If $\rho=1$ and $\left(a_{1}\right)$ and $\left(a_{1}+1\right)$ are eligible 1-tuples, then $T_{1,1} g_{a_{1}, 1} \equiv y^{\sigma_{1}} g_{a_{1}+1,1}$.

(f) If $\rho=1$ and $1 \leq j \leq r(\emptyset)$, then $T_{1, j} g_{f(\emptyset), 1} \equiv y^{\sigma_{1}+1-r(\emptyset)} f_{j}$.

(g) The ideals $g K^{(n)}$ and $y^{n} L A$ of $A$ are equal.

Proof. We have

$$
h_{a_{1}, 0}=x^{n-a_{1} \sigma_{1}} \sum_{p+q=a_{1}-1} T_{1,1}^{p} T_{1, \sigma_{1}+1}^{q} \pi_{1, p \sigma_{1}}+T_{1,1}^{a_{1}} \Delta_{a_{1} \sigma_{1}} .
$$

The facts

$$
\begin{aligned}
x^{\sigma_{1}} T_{1,1} & \equiv y^{\sigma_{1}} T_{1, \sigma_{1}+1}, \\
\Delta_{a_{1} \sigma_{1}} & =x^{n-\left(a_{1}+1\right) \sigma_{1}+1} \Delta_{a_{1} \sigma_{1}, \sigma_{1}-1}+y^{\sigma_{1}} \Delta_{\left(a_{1}+1\right) \sigma_{1}}, \text { and } \\
T_{1,1} x \Delta_{a_{1} \sigma_{1}, \sigma_{1}-1} & \equiv y^{\sigma_{1}} \pi_{1, a_{1} \sigma_{1}}
\end{aligned}
$$

may be found in Observation 3.8, Remark 3.4.b, and Observation 3.9, respectively. These facts establish (a). The same type of methods are used to prove (b). One uses $x^{\sigma_{2}} T_{2,1} \equiv y^{\sigma_{2}} T_{2, \sigma_{2}+1}$ in the first two summands of $T_{2,1} h_{a_{1}, a_{2}}$. In the third summand one uses

$$
\Delta_{a_{1} \sigma_{1}+a_{2} \sigma_{2}}=x^{n-a_{1} \sigma_{1}-\left(a_{2}-1\right) \sigma_{2}} x \Delta_{a_{1} \sigma_{1}+a_{2} \sigma_{2}, \sigma_{2}-1}+y^{\sigma_{2}} \Delta_{a_{1} \sigma_{1}+\left(a_{2}+1\right) \sigma_{2}} .
$$

Once again, Observation 3.9 yields

$$
T_{2,1} x \Delta_{a_{1} \sigma_{1}+a_{2} \sigma_{2}, \sigma_{2}-1}(x, y)=y^{\sigma_{2}} \pi_{2, a_{1} \sigma_{1}+a_{2} \sigma_{2}} .
$$

We prove (c). Notice that

$$
n-a_{1} \sigma_{1}-f\left(a_{1}\right) \sigma_{2}=\sigma_{2}-r\left(a_{1}\right)+1
$$


hence Observations 3.8 and 3.9 yield

$$
\begin{aligned}
T_{2, j} x^{n-a_{1} \sigma_{1}-f\left(a_{1}\right) \sigma_{2}} & \equiv T_{2, j+\sigma_{2}-r\left(a_{1}\right)+1} y^{\sigma_{2}-r\left(a_{1}\right)+1} \text { and } \\
T_{2, j} \Delta_{a_{1} \sigma_{1}+f\left(a_{1}\right) \sigma_{2}} & \equiv y^{\sigma_{2}+1-r\left(a_{1}\right)} \pi_{2, \sigma_{2}+1-r\left(a_{1}\right), j}^{\prime} .
\end{aligned}
$$

The proof of (d) is similar. The equality

$$
n-f(\emptyset) \sigma_{1}=\sigma_{1}+1-r(\emptyset)
$$

implies

$$
\begin{aligned}
T_{1, j} x^{n-f(\emptyset) \sigma_{1}} & \equiv y^{\sigma_{1}+1-r(\emptyset)} T_{1, \sigma_{1}+1-r(\emptyset)+j} \text { and } \\
T_{1, j} \Delta_{f(\emptyset) \sigma_{1}} & \equiv y^{\sigma_{1}+1-r(\emptyset)} \pi_{1, \sigma_{1}+1-r(\emptyset), j}^{\prime} .
\end{aligned}
$$

When $\rho=1$, we have $\sigma_{2}=1, T_{2,1}=y, T_{2,2}=x, a_{1} \sigma_{1}+f\left(a_{1}\right)=n-1$, and $r\left(a_{1}\right)=1$. We quickly calculate

$$
\pi_{2,1,1}^{\prime}=c_{n-1} x+c_{n} y \quad \text { and } \quad \pi_{2, a_{1} \sigma_{1}+1}=c_{a_{1} \sigma_{1}+p} x .
$$

We now have

$$
\begin{gathered}
x \sum_{p+q=f\left(a_{1}\right)-1} y^{p} x^{q} \pi_{2, a_{1} \sigma_{1}+p}+y^{f\left(a_{1}\right)} \pi_{2,1,1}^{\prime}=\Delta_{a_{1} \sigma_{1}} \text { and } \\
g_{a_{1}, 1}=x^{n-a_{1} \sigma_{1}} \sum_{p+q=a_{1}-1} T_{1,1}^{p} T_{1, \sigma_{1}+1}^{q} \pi_{1, p \sigma_{1}}+T_{1,1}^{a_{1}} \Delta_{a_{1} \sigma_{1}} .
\end{gathered}
$$

The arguments of (a) and (d) establish (e) and (f), respectively.

We prove $(\mathrm{g})$ by showing that

$$
g T^{\boldsymbol{a}} T_{k+1,1}^{f(a)} T_{k+1, j} \equiv y^{n} G_{(\boldsymbol{a}, j)},
$$

whenever $\boldsymbol{a}$ is an eligible $k$-tuple and $1 \leq j \leq r(\boldsymbol{a})$. Start with $\rho=2$. Recall that $g=h_{0,0}$ and $T_{3,1}=y$. If $\boldsymbol{a}=\left(a_{1}, a_{2}\right)$ is an eligible tuple and $j=1$, then (a) and (b) show that

$$
g T_{1,1}^{a_{1}} T_{2,1}^{a_{2}} T_{3,1}^{f(\boldsymbol{a})} T_{3, j}=y^{a_{1} \sigma_{1}+a_{2} \sigma_{2}+f(\boldsymbol{a})+1} h_{a_{1}, a_{2}}=y^{n} G_{(\boldsymbol{a}, 1)} .
$$

If $\left(a_{1}\right)$ is an eligible tuple and $1 \leq j \leq r\left(a_{1}\right)$, then (a), (b), and (c) yield

$$
g T_{1,1}^{a_{1}} T_{2,1}^{f\left(a_{1}\right)} T_{2, j}=y^{a_{1} \sigma_{1}+f\left(a_{1}\right) \sigma_{2}+\sigma_{2}+1-r\left(a_{1}\right)} g_{a_{1}, j}=y^{n} G_{\left(\left(a_{1}\right), 1\right)} .
$$


If $1 \leq j \leq r(\emptyset)$, then (a) and (d) yield

$$
g T_{1,1}^{f(\emptyset)} T_{1, j}=y^{f(\emptyset) \sigma_{1}+\sigma_{1}+1-r(\emptyset)} f_{j}=y^{n} G_{(\emptyset, 1)} .
$$

Now take $\rho=1$. Recall that $g=g_{0,1}$ and $y=T_{2,1}$. If $\left(a_{1}\right)$ is an eligible tuple and $1 \leq j \leq r\left(a_{1}\right)$, then $r\left(a_{1}\right)=1=j$ and (e) gives

$$
g T_{1,1}^{a_{1}} T_{2,1}^{f\left(a_{1}\right)} T_{2, j}=y^{a_{1} \sigma_{1}+f\left(a_{1}\right)+1} g_{a_{1}, 1}=y^{n} G_{\left.\left(a_{1}\right), j\right)} .
$$

Finally, if $1 \leq j \leq r(\emptyset)$, then (e) and (f) give

$$
g T_{1,1}^{f(\emptyset)} T_{1, j}=y^{f(\emptyset) \sigma_{1}+\sigma_{1}+1-r(\emptyset)} f_{j}=y^{n} G_{(\emptyset, j)} .
$$

\section{Depth, reduction number, regularity, and Hilbert function.}

This section is mainly about the fiber ring $\mathcal{F}(I)=\mathcal{R}(I) /(x, y)$. We compute the depth, reduction number, and regularity of $\mathcal{F}(I)$. A related invariant, the postulation number of $\mathcal{F}(I)$, is computed in Corollary 6.9. Most of the results are collected in Theorem 4.2; these results are proved, in a more general setting, in [22]; see Theorem 4.3. The main result of this section is Theorem 4.4 where we calculate the reduction number, $r(I)$, of $I$ when $\rho=2$. Observation 4.1 shows how we will use the rational normal scrolls of Section 1 to calculate $r(I)$. Theorem 4.5 is a general result connecting reduction number and Hilbert function for rings of minimal multiplicity; it is based on the Socle Lemma of Huneke-Ulrich. Proposition 4.7 is a curious result which allows us to circumvent the characteristic zero hypothesis in the Socle Lemma; we create a ring in which the bracket powers of the maximal ideal are equal to the ordinary powers, independent of the characteristic of the field.

A reduction of an ideal $I$ is an ideal $J \subseteq I$ with $J I^{i}=I^{i+1}$ for all large $i$. The reduction number of $I$ with respect to the reduction $J$ is

$$
r_{J}(I)=\min \left\{i \geq 0 \mid J I^{i}=I^{i+1}\right\} .
$$

A reduction $J$ of $I$ is minimal if $J$ does not contain any other reduction of $I$. The reduction number of $I$ is defined by

$$
r(I)=\min \left\{r_{J}(I) \mid J \text { is a minimal reduction of } I\right\} .
$$

Let $B=\oplus_{i \geq 0} B_{i}$ be a standard graded algebra over an infinite field $k$ with $D$ equal to the Krull dimension of $B$. The unique maximal homogenous ideal of $B$ is denoted by $\mathfrak{m}_{B}$. Every minimal reduction of $\mathfrak{m}_{B}$ is generated by $D$ linear forms in $\mathfrak{m}_{B}$. Sometimes it is convenient to write $r(B)$ in place of $r\left(\mathfrak{m}_{B}\right)$. The reduction number of an ideal $I$ is equal to be the reduction number of the maximal ideal in the fiber ring $\mathcal{F}(I)$; that is $r(I)=r(\mathcal{F}(I))$. 
Observation 4.1. Adopt the notation of Definition 1.5. In light of Observation 2.8, we may assume that $y^{n} T_{1}$ appears in $g$ with a non-zero coefficient. Let ${ }^{-}$mean "image in $A^{-}=A /(x, y) A "$. The following statements hold.

(1) The ring $A^{-}$is defined by the maximal minors of a scroll matrix and the ideal $\left(K^{(n)}\right)^{\sim}$ is the $n^{\text {th }}$ symbolic power of a height one prime ideal of $A^{\sim}$.

(2) The fiber ring of $I$ is equal to $\mathcal{F}(I)=A^{\sim} / \mathcal{A}^{\sim}$.

(3) The ideals $\mathcal{A}^{\smile}$ and $\left(K^{(n)}\right)^{\sim}(-1)$ of $A^{\smile}$ are isomorphic.

(4) The reduction number of $I$ is equal to $r(I)=r\left(A^{\smile} /\left(K^{(n)}\right)^{\smile}\right)+1$.

Proof. Assertion (1) is essentially obvious. The ring $\mathcal{A}^{\llcorner}$equals $k\left[T_{1}, \ldots, T_{m}\right] / I_{2}\left(\psi^{\smile}\right)$ for

$$
\psi^{\sim}= \begin{cases}{\left[\begin{array}{ll}
\psi_{1} & \psi_{2}
\end{array}\right],} & \text { if } \rho=2, \\
\psi_{1}, & \text { if } \rho=1 .\end{cases}
$$

The ideal $\left(K^{(n)}\right)^{`}$ of $A^{`}$ is equal to

$$
\begin{cases}T_{1,1}^{f(\emptyset)}\left(T_{1,1}, \ldots T_{1, r(\emptyset)}\right), & \text { if } \rho=1 \\ T_{1,1}^{f(\emptyset)}\left(T_{1,1}, \ldots T_{1, r(\emptyset)}\right)+\sum_{a_{1}=0}^{f(\emptyset)} T_{1,1}^{a_{1}} T_{2,1}^{f\left(a_{1}\right)}\left(T_{2,1}, \ldots T_{2, r\left(a_{1}\right)}\right), & \text { if } \rho=2\end{cases}
$$

and therefore, $\left(K^{(n)}\right)^{\sim}$ is the $n^{\text {th }}$ symbolic power of the ideal of $A^{\sim}$ which is generated by the top row of $\psi^{`}$. For $(2)$, we have $\mathcal{F}(I)=\mathcal{R}(I) /(x, y) \mathcal{R}(I)$ and $\mathcal{R}(I)=A / \mathcal{A}$.

We prove (3). Recall that $g=g(x, y)=\sum_{i=0}^{n} c_{i} x^{n-i} y^{i}$. Recall also that the ideals

$$
y^{n} \mathcal{A} A \text { and } g(x, y) K^{(n)}
$$

of $A$ are equal. Fix a pair of subscripts $i, j$ with $1 \leq i \leq \rho$ and $1 \leq j \leq \sigma_{i}$. Multiply both sides by $T_{i, j}^{n}$. Notice that, in $A$,

$$
T_{i, j}^{n} g=\sum_{i=0}^{n} c_{i}\left(T_{i, j} x\right)^{n-i}\left(T_{i, j} y\right)^{i}=\sum_{i=0}^{n} c_{i}\left(T_{i, j+1} y\right)^{n-i}\left(T_{i, j} y\right)^{i}=y^{n} g\left(T_{i, j+1}, T_{i, j}\right) .
$$

Conclude that

$$
y^{n} T_{i, j}^{n} \mathcal{A} A=y^{n} g\left(T_{i, j+1}, T_{i, j}\right) K^{(n)} .
$$

The ring $A$ is a domain and $y \neq 0$; so,

$$
T_{i, j}^{n} \mathcal{A} A=g\left(T_{i, j+1}, T_{i, j}\right) K^{(n)} .
$$


The equality

$$
T_{1,1}^{n} \mathcal{A}^{\smile}=g\left(T_{1,2}, T_{1,1}\right)\left(K^{(n)}\right)^{\smile}
$$

is especially interesting to us because we have arranged the data in order to know that the monomial $T_{1,1}^{n+1}$ appears in $g\left(T_{1,2}, T_{1,1}\right)$ with a non-zero coefficient. The argument used to prove (1.12) shows that $g\left(T_{1,2}, T_{1,1}\right)$ is not zero in the domain $A^{2}$. The elements $T_{1,1}^{n}$ and $g\left(T_{1,2}, T_{1,1}\right)$ both are regular on $A^{\sim}$. Assertion (3) follows.

We prove (4). We have seen that

$$
r(I)=r(\mathcal{F}(I))=r\left(A^{`} / \mathcal{A}^{`}\right) .
$$

The reduction numbers of the two-dimensional standard graded rings $A^{\sim} / \mathcal{A}^{\iota}$ and $A^{\sim} /\left(K^{(n)}\right)^{`}$ may be computed by reducing modulo two generic linear forms, see, for example, [26] or [27]. Let $k(u)$ be the appropriate purely transcendental extension of $k$, let $\ell_{1}$ and $\ell_{2}$ be two generic linear forms in $A^{`} \otimes_{k} k(u)$, and let - represent image in $\overline{A^{\sim}}=\left(A^{\sim} \otimes_{k} k(u)\right) /\left(\ell_{1}, \ell_{2}\right)\left(A^{\sim} \otimes_{k} k(u)\right)$. The ideals $\overline{\mathcal{A}^{\sim}}$ and $\overline{\left(K^{(n)}\right)^{\sim}}(-1)$ of $\overline{A^{\sim}}$ are isomorphic because $z, \ell_{1}, \ell_{2}$ is a regular sequence in $A^{\sim} \otimes_{k} k(u)$ for every non-zero element $z$ in $A^{\sim}$. We know, see for example Proposition 5.2, that $\lambda\left({\overline{A^{\sim}}}_{s}\right)=m-2$ for all $s \geq 1$; so,

$$
\begin{aligned}
& \left(\overline{A^{`}} / \overline{\mathcal{A}^{\ulcorner}}\right)_{s}=0 \Longleftrightarrow \lambda\left(\overline{\mathcal{A}^{\ulcorner}}\right)_{s}=m-2 \Longleftrightarrow \lambda\left(\overline{\left(K^{(n)}\right)^{\ulcorner}}\right)_{s-1}=m-2 \\
& \Longleftrightarrow\left(\overline{A^{\sim}} / \overline{\left(K^{(n)}\right)^{`}}\right)_{s-1}=0, \quad \text { and } \\
& r(I)=r\left(A^{\sim} / \mathcal{A}^{\curlyvee}\right)=\max \left\{s \mid\left(\overline{A^{\smile}} / \overline{\mathcal{A}^{\ulcorner}}\right)_{s} \neq 0\right\}
\end{aligned}
$$

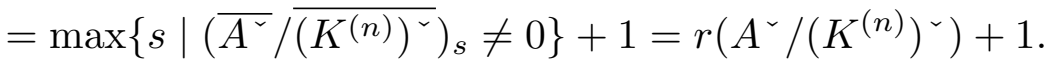

Theorem 4.2. Adopt the notation of Definition 1.5 with $n \geq 2$.

(a) We have

$$
\operatorname{depth} \operatorname{gr}_{I}(R)+1=\operatorname{depth} \mathcal{R}(I)=\operatorname{depth} \mathcal{F}(I)= \begin{cases}2, & \text { if } \rho=1 \\ 1, & \text { if } \rho=2\end{cases}
$$

In particular, $\mathcal{F}(I)$ is Cohen-Macaulay if and only if $\rho=1$.

(b) If $\rho=1$, then

$$
r(I)=r\left(\mathfrak{m}_{\mathcal{F}(I)}\right)=\operatorname{reg} \mathcal{F}(I)=\left\lceil\frac{n-1}{\sigma_{1}}\right\rceil+1
$$


(c) If $\rho=2$, then $\operatorname{reg}(\mathcal{F}(I))=\left\lceil\frac{n-1}{\sigma_{2}}\right\rceil+1$.

Remark. The value of $r(I)=r\left(\mathfrak{m}_{\mathcal{F}(I)}\right)$ when $\rho=2$ is computed in Theorem 4.4.

Proof. We apply Theorem 1.11 and Observation 4.1. The isomorphisms

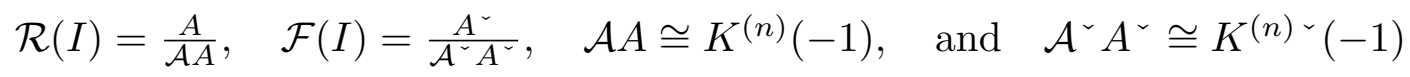

tell us that

$$
\begin{gathered}
\operatorname{depth} \mathcal{R}(I)=\operatorname{depth} A / K^{(n)}, \quad \operatorname{depth} \mathcal{F}(I)=\operatorname{depth} A^{\smile} /\left(K^{(n)}\right)^{\smile}, \quad \text { and } \\
\operatorname{reg} \mathcal{F}(I)=\operatorname{reg}\left(A^{\smile} / \mathcal{A}^{\smile} A\right)=\operatorname{reg}\left(\mathcal{A}^{\smile} A\right)-1=\operatorname{reg} K^{(n)} .
\end{gathered}
$$

One may now read $\operatorname{reg} \mathcal{F}(I)=\left\lceil\frac{n-1}{\sigma_{\rho}}\right\rceil+1$ from Theorem 4.3. If $\rho=2$, then one may also read $\operatorname{depth} \mathcal{R}(I)=\operatorname{depth} \mathcal{F}(I)=1$. If $\rho=1$, then the variable $T_{m}$ is not involved in

$$
\psi=\left[\begin{array}{llll}
T_{1} & \ldots & T_{m-2} & y \\
T_{2} & \ldots & T_{m-1} & x
\end{array}\right], \quad \psi^{\llcorner}=\left[\begin{array}{lll}
T_{1} & \ldots & T_{m-2} \\
T_{2} & \ldots & T_{m-1}
\end{array}\right],
$$

$K^{(n)}$, or $K^{(n)}$ ` ; so $T_{m}$ is regular on $A / K^{(n)}$ and $A^{\smile} /\left(K^{(n)}\right)^{`}$ and Theorem 4.3 tells us that

$$
\operatorname{depth} A /\left(T_{m}, K^{(n)}\right)=\operatorname{depth} A^{\sim} /\left(T_{m},\left(K^{(n)}\right)^{\smile}\right)=1 .
$$

Since $\mathcal{R}(I)$ is not Cohen-Macaulay, it follows that $\operatorname{gr}_{I}(R)$ is not Cohen-Macaulay either by [25] and then $\operatorname{depth} \mathcal{R}(I)=\operatorname{depth} \operatorname{gr}_{I}(R)+1$ by [17]. Finally, we recall that if $\mathcal{F}(I)$ is a Cohen-Macaulay ring, then reg $\mathcal{F}(I)=r(I)$; indeed both quantities are equal to the top socle degree of $\mathcal{F}(I)$ modulo a linear system of parameters.

Theorem 4.3. Let $\sigma_{1} \geq \cdots \geq \sigma_{\ell} \geq 1$ and $n \geq 2$ be integers, and let $P$ be the polynomial ring

$$
k\left[\left\{T_{i, j} \mid 1 \leq i \leq \ell \quad \text { and } \quad 1 \leq j \leq \sigma_{i}+1\right\}\right] .
$$

For each $u$, with $1 \leq u \leq \ell$, let $\psi_{u}$ be the generic scroll matrix

$$
\psi_{u}=\left[\begin{array}{ccccc}
T_{u, 1} & T_{u, 2} & \ldots & T_{u, \sigma_{u}-1} & T_{u, \sigma_{u}} \\
T_{u, 2} & T_{u, 3} & \ldots & T_{u, \sigma_{u}} & T_{u, \sigma_{u}+1}
\end{array}\right] .
$$

Let $\Psi$ be the matrix

$$
\Psi=\left[\begin{array}{lllll}
\psi_{1} & \mid & \ldots & \mid & \psi_{\ell}
\end{array}\right],
$$

$A$ be the ring $P / I_{2}(\Psi)$, and $K$ be the ideal in $A$ generated by the entries of the top row of $\Psi$. Then

$$
\operatorname{depth} A / K^{(n)}=1 \quad \text { and } \quad \operatorname{reg} K^{(n)}=\left\lceil\frac{n-1}{\sigma_{\ell}}\right\rceil+1 .
$$

Proof. See [22]. 
red1 Theorem 4.4. If I is as in Definition 1.5 with $\rho=2$, then the following statements hold.

(a) $\left\lceil\frac{n}{\sigma_{1}}\right\rceil \leq r(I) \leq\left\lceil\frac{n-1}{\sigma_{1}}\right\rceil+1$.

(b) If $\sigma_{1} \mid n-1$, then $r(I)=\left\lceil\frac{n}{\sigma_{1}}\right\rceil=\left\lceil\frac{n-1}{\sigma_{1}}\right\rceil+1$.

(c) $r(I)=\left\lceil\frac{n}{\sigma_{1}}\right\rceil \Longleftrightarrow \mathrm{H}_{\left(K^{(n)}\right)^{-}}\left(\left\lceil\frac{n}{\sigma_{1}}\right\rceil\right) \geq m-2$.

Remarks. 1. The exact value of $\mathrm{H}_{\left(K^{(n)}\right)^{-}}\left(\left\lceil\frac{n}{\sigma_{1}}\right\rceil\right)$ depends on the interaction between the three integers $\sigma_{1}, \sigma_{2}$, and $n$, and is not difficult to calculate. One simply counts the number of monomials of degree $\left\lceil\frac{n}{\sigma_{1}}\right\rceil=f(\emptyset)+1$ that appear in the minimal generating set for $\left(K^{(n)}\right)^{`}$ listed in the proof of Observation 4.1.

2. We prove (a) now. Assertion (b) is obvious.

3. Part (a) shows that there are only two possible choices for $r(I)$. Furthermore, in the proof of (a), we learn a necessary condition for $r(I)$ to take on the smaller of the two values; namely, that $\left(K^{(n)}\right)^{-}$contain at least $m-2$ linearly independent homogeneous elements of degree $\left\lceil\frac{n}{\sigma_{1}}\right\rceil$. The proof that this condition is sufficient (i.e., part (c)) appears at the end of this section.

Proof of (a). Adopt the notation of Observation 4.1. So, $A^{\sim}=k\left[T_{1}, \ldots T_{m}\right] / I_{2}\left(\psi^{\sim}\right)$ and $\mathcal{F}(I)=A^{\smile} / \mathcal{A}^{\smile}$. The convention of (1.4) is in effect and each variable $T_{i}$ has two names.

We first establish the inequality on the right. Let $J=\left(T_{m}, T_{\sigma_{1}+1}-T_{\sigma_{1}+2}\right)$ and write ${ }^{-}$for images in $\overline{A^{`}}=A^{\sim} / J A^{\llcorner}$. Notice that $T_{m}, T_{\sigma_{1}+1}-T_{\sigma_{1}+2}$ form a linear system of parameters in $A^{\sim} /\left(K^{(n)}\right)^{\sim}$; hence,

$$
\begin{aligned}
r(I)=r\left(A^{\smile} /\left(K^{(n)}\right)^{\smile}\right)+1 & \leq \max \left\{i \mid\left[\overline{A^{\smile}} \overline{\left(K^{(n)}\right)^{`}}\right]_{i} \neq 0\right\}+1 \\
& \left.=\max \left\{i \mid \lambda\left(\overline{\left(\left(K^{(n)}\right)^{`}\right.}\right)_{i}\right)<m-2\right\}+1 .
\end{aligned}
$$

The generators of $\overline{\left(K^{(n)}\right)^{`}}$ are listed in the proof of Observation 4.1. Observe that $T_{2,1} T_{2, j}=0$ in $\overline{A^{\ulcorner}}$, for $1 \leq j \leq \sigma_{2}+1$, because

$$
T_{2,1} T_{2, j}=T_{1, \sigma_{1}+1} T_{2, j}=T_{1, \sigma_{1}-\sigma_{2}+j} T_{2, \sigma_{2}+1}=0 .
$$

Notice that all variables make sense. The first and last equations are due to $J$. The middle equation always happens. So,

$$
\overline{\left(K^{(n)}\right)^{\smile}}=T_{1,1}^{f(\emptyset)}\left[\left(T_{1,1}, \ldots, T_{1, r(\emptyset)}\right)+\chi(f(f(\emptyset))=0)\left(T_{2,1}, \ldots, T_{2, r(f(\emptyset))}\right)\right] .
$$


Observe immediately that $\left(\overline{\left(K^{(n)}\right)^{`}}\right)_{i}=0$ for $i<f(\emptyset)+1=\left\lceil\frac{n}{\sigma_{1}}\right\rceil$ and

$$
\left(\overline{A^{`}}\right)_{i}=T_{1,1}^{i-1}{\overline{A^{`}}}_{1} \subseteq \overline{\left(K^{(n)}\right)^{`}}
$$

for $i>f(\emptyset)+1$. Recall that

$$
r(\emptyset)=\sigma_{1} \Longleftrightarrow \sigma_{1} \mid(n-1) \Longleftrightarrow f(\emptyset) \sigma_{1}=n-1 .
$$

We now see that

$$
\left.r(I)-1 \leq \max \left\{i \mid \lambda\left(\overline{\left(\bar{K}^{(n)}\right)^{`}}\right)_{i}\right)<m-2\right\}= \begin{cases}f(\emptyset), & \text { if } \sigma_{1} \mid(n-1), \\ f(\emptyset)+1, & \text { if } \sigma_{1} \chi(n-1) ;\end{cases}
$$

hence, $r(I)-1 \leq\left\lceil\frac{n-1}{\sigma_{1}}\right\rceil$.

Now we establish the inequality on the left. Let $\ell_{1}$ and $\ell_{2}$ be two general linear forms in $k\left[T_{1}, \ldots, T_{m}\right], J$ be the ideal $\left(\ell_{1}, \ell_{2}\right)$ of $A^{\sim}$, and - represent image in $\overline{A^{\sim}}=A^{\sim} / J A^{\llcorner}$. We see that $\ell_{1}, \ell_{2}$ forms a general linear system of parameters in $A^{\smile} /\left(K^{(n)}\right)^{\sim}$; hence,

$$
f(\emptyset) \leq \max \left\{i \mid \lambda\left(\left(\overline{\left(K^{(n)}\right)^{\smile}}\right)_{i}\right)<m-2\right\}=r\left(A^{\smile} /\left(K^{(n)}\right)^{\smile}\right)=r(I)-1
$$

and $\left\lceil\frac{n}{\sigma_{1}}\right\rceil=f(\emptyset)+1 \leq r(I)$.

The proof of Theorem 4.4(c) will be based in the next general result relating reduction numbers to Hilbert functions. Assume that $(A, \mathfrak{m})$ is a one-dimensional Cohen-Macaulay standard graded ring over a field with minimal multiplicity $e$, and $L$ is a homogeneous $m$-primary ideal. In this case it is obvious that $r(A / L)<s$ if and only if $\mathrm{H}_{L}(s) \geq e$. The purpose of the following theorem is to prove an analogous statement in dimension two. The first difference function of the Hilbert function is denoted $\Delta \mathrm{H}$ and is defined by $\Delta \mathrm{H}_{M}(i)=\mathrm{H}_{M}(i)-\mathrm{H}_{M}(i-1)$.

SOc

Theorem 4.5. Let $(A, \mathfrak{m})$ be a standard graded domain over a field $k$. Assume that $A$ is a two-dimensional Cohen-Macaulay ring with minimal multiplicity e. Let $s$ be a positive integer and $L$ be a homogeneous ideal of $A$ with $\operatorname{dim} A / L=1$. Let $\ell$ be a generic linear form defined over a purely transcendental extension field $k^{\prime}$ of $k$ and assume that, after making a possible further field extension $k^{\prime \prime}$ of $k^{\prime}$,

$$
\mathfrak{m}^{s} \subset\left(\left\{y^{s} \mid y \in A_{1} \otimes_{k} k^{\prime \prime}\right\}\right)+(L, \ell) .
$$

One has

$$
r(A / L)<s \Longleftrightarrow\left(\Delta \mathrm{H}_{L}\right)(s) \geq e .
$$


Remark. The hypothesis (4.6) is automatically satisfied if the characteristic of $k$ is zero.

Proof. We replace $A$ by $A \otimes_{k} k^{\prime}$. This does not change the reduction number of $A / L$ according to [26]. Write ${ }^{-}$for images in $\bar{A}=A /(\ell)$. Notice that $\bar{A}$ is a domain by [15] and $r(A / L)=r(\bar{A} / \bar{L})$ again by [26], whereas $\Delta \mathrm{H}_{L}=\mathrm{H}_{L / \ell L}$. Moreover, $\bar{A} / \bar{L}$ is an Artinian ring. We need to show that $\bar{L}_{s}=\bar{A}_{s}$ if and only if $\mathrm{H}_{L / \ell L}(s) \geq e$. Since $\mathrm{H}_{\bar{A}}(s)=e$ it remains to prove that $\mathrm{H}_{\bar{L}}(s) \geq e$ if and only if $\mathrm{H}_{L / \ell L}(s) \geq e$. As $\mathrm{H}_{\bar{L}}(s) \leq \mathrm{H}_{L / \ell L}(s)$ it suffices to show that if $\mathrm{H}_{L / \ell L}(s) \geq e$ then $\mathrm{H}_{\bar{L}}(s) \geq e$.

If $\bar{A} / \bar{L}$ has a nonzero homogeneous socle element of degree $j<s$, then $z \overline{\mathfrak{m}} \subset \bar{L}$ for some homogeneous nonzero element $z \in \bar{A}$ of degree $j$. Hence $\mathrm{H}_{\bar{L}}(s) \geq \mathrm{H}_{z \overline{\mathfrak{m}}}(s)=$ $\mathrm{H}_{\overline{\mathfrak{m}}}(s-j)$ because $\bar{A}$ is a domain. Clearly $\mathrm{H}_{\overline{\mathfrak{m}}}(s-j)=e$ since $s-j \geq 1$, which gives $\mathrm{H}_{\bar{L}}(s) \geq e$.

Thus we may assume that the socle of $\bar{A} / \bar{L}$ is concentrated in degrees $\geq s$. For the remainder of the proof we do not need anymore that $\bar{A}$ is a domain, thus we may extend the ground field to assume that condition (4.6) holds. We wish to apply the Socle Lemma [18,Cor. 3.11(i)] to the exact sequence

$$
0 \longrightarrow(L: \ell / L)(-1) \longrightarrow A / L(-1) \longrightarrow A / L \longrightarrow A /(L, \ell)=\bar{A} / \bar{L} \longrightarrow 0 .
$$

The statement of the Socle Lemma requires that the field have characteristic zero; however, this hypothesis is only used in order to ensure that condition (4.6) is satisfied. Also, the Socle Lemma requires $\ell$ to be a general linear form, but the proof also works for generic linear forms, see [18, Prop. 3.5]. So the Socle Lemma may be applied in the present situation. In our setting it says that if the socle of $\bar{A} / \bar{L}$ is concentrated in degrees $\geq s$ then so is $L: \ell / L$. Thus we can write $L: \ell=L+K$ with $K$ a homogeneous $A$-ideal concentrated in degrees $\geq s$. It follows that $L \cap(\ell)=\ell(L: \ell)=\ell L+\ell K$. Therefore $[L \cap(\ell)]_{s}=[\ell L]_{s}$, which gives $[\bar{L}]_{s}=[L /(\ell L)]_{s}$. Thus $\mathrm{H}_{\bar{L}}(s)=\mathrm{H}_{L / \ell L}(s) \geq e$.

In the next proposition we show that the homogeneous coordinate ring of any rational normal curve satisfies assumption (4.6) regardless of the characteristic. If one is only interested in characteristic zero, then Proposition 4.7 may be skipped.

P39.2 Proposition 4.7. Let $k^{\prime}$ be the field of rational functions $k\left(\lambda_{1}, \ldots, \lambda_{c}\right)$ in $c$ variables over the field $k$ and let $k^{\prime \prime}$ be any extension field of $k^{\prime}$ for which the polynomial

$$
P(x)=x^{c}-\lambda_{c} x^{c-1}-\cdots-\lambda_{2} x-\lambda_{1}
$$

splits into linear factors. Let $A$ be the standard graded algebra $k^{\prime \prime}\left[T_{1}, \ldots, T_{c}\right] / I_{2}(\psi)$, where $\psi$ is the scroll matrix

$$
\psi=\left[\begin{array}{ccccc}
T_{1} & T_{2} & \ldots & T_{c-1} & T_{c} \\
T_{2} & T_{3} & \ldots & T_{c} & \sum_{i=1}^{c} \lambda_{i} T_{i}
\end{array}\right] .
$$


Then there exist homogeneous linear forms $v_{1}, \ldots, v_{c}$ in $A_{1}$ such that $v_{1}^{s}, \ldots, v_{c}^{s}$ is a $k^{\prime \prime}$-basis for $A_{s}$ for all $s \geq 1$.

Proof. Recall that $A$ is a one-dimensional standard graded ring of minimal multiplicity and $T_{1}$ is an $A$-regular linear form. (Some readers will find Proposition 5.2 to be helpful at this point.) Hence for any $s \geq 1$ one has $\operatorname{dim} A_{s}=c$ and $T_{1}^{s-1} T_{1}, \ldots, T_{1}^{s-1} T_{c}$ form a basis of $A_{s}$. Once we have identified suitable candidates for $v_{1}, \ldots, v_{c}$, then we need only verify that $v_{1}^{s}, \ldots, v_{c}^{s}$ are linearly independent. Ultimately, we pick $v_{1}, \ldots, v_{c}$ to be a basis for $A_{1}$ which yields a simultaneous diagonalization of all of the linear transformations $\varphi_{j}=T_{1}^{-1} T_{j}: A_{1} \rightarrow A_{1}$.

Let $k^{\prime \prime \prime} \subseteq k^{\prime \prime}$ be the splitting field of $P(x)$ over $k^{\prime}$. Since $\lambda_{1}, \ldots, \lambda_{c}$ are variables over $k$, the polynomial $P(x)$ has $c$ distinct roots. In particular, the field extension $k^{\prime} \subset k^{\prime \prime \prime}$ is separable.

The matrix representation of the endomorphism $\varphi_{2}=T_{1}^{-1} T_{2}: A_{1} \rightarrow A_{1}$ with respect to the basis $T_{1}, \ldots, T_{c}$ is

$$
\left[\begin{array}{ccccccc}
0 & & & & & & \lambda_{1} \\
1 & 0 & \cdot & & & & \cdot \\
& 1 & \cdot & \cdot & & & \cdot \\
& & & \cdot & \cdot & & \cdot \\
& & & & \cdot & 0 & \cdot \\
& & & & & 1 & \lambda_{c}
\end{array}\right] .
$$

This being a companion matrix it follows that the minimal polynomial of $\varphi_{2}$ is $P(x)$, which has $c$ distinct roots in $k^{\prime \prime \prime}$. Thus $\varphi_{2}$ is diagonalizable over $k^{\prime \prime \prime}$ with eigenvectors, say, $v_{1}, \ldots, v_{c}$.

On the other hand, for $2 \leq j \leq c$ one has $T_{1} T_{j}=T_{2} T_{j-1}$, hence $\varphi_{j}=\varphi_{2} \varphi_{j-1}$. Thus one sees by induction on $j$ that $v_{1}, \ldots, v_{c}$ are eigenvectors for every $\varphi_{j}$. In other words, there exist $\alpha_{i} \in k^{\prime \prime \prime}$ with

$$
T_{j} v_{i}=\alpha_{i}^{j-1} T_{1} v_{i} .
$$

Thus, $\mathfrak{m} v_{i} \subset A T_{1} v_{i}$ for $1 \leq i \leq c$, and then $\mathfrak{m}^{s-1} v_{i} \subset A T_{1}^{s-1} v_{i}$. In particular, $v_{i}^{s} \in A T_{1}^{s-1} v_{i}$, say

$$
v_{i}^{s}=\beta_{i} T_{1}^{s-1} v_{i} .
$$

for some $\beta_{i} \in k^{\prime \prime \prime}$.

Recall that $k^{\prime}\left[T_{1}, \ldots, T_{c}\right] / I_{2}(\Psi)$ is a domain and $k^{\prime} \subset k^{\prime \prime \prime}$ is a separable field extension. Therefore $A$ is reduced, hence $v_{i}^{s} \neq 0$, which gives $\beta_{i} \neq 0$. Since $T_{1}^{s-1}$ is a non zerodivisor and $\beta_{i}$ are nonzero scalars, (4.8) shows that $v_{1}^{s}, \ldots, v_{c}^{s}$ are indeed linearly independent over $k^{\prime \prime \prime}$ and therefore also over $k^{\prime \prime}$. 
Proof of part (c) of Theorem 4.4. Start with the ring $A^{\vee}$ and the ideal $\left(K^{(n)}\right)^{`}$ of Observation 4.1. From Theorem 4.4 (a) and Observation 4.1 we know that

$$
\left\lceil\frac{n}{\sigma_{1}}\right\rceil \leq r(I)=r\left(A^{\smile} /\left(K^{(n)}\right)^{\smile}\right)+1 .
$$

Hence it suffices to show that

$$
r\left(A^{\sim} /\left(K^{(n)}\right)^{\smile}\right)<\left\lceil\frac{n}{\sigma_{1}}\right\rceil \Longleftrightarrow \mathrm{H}_{\left(K^{(n)}\right)^{\sim}}\left(\left\lceil\frac{n}{\sigma_{1}}\right\rceil\right) \geq m-2 .
$$

First assume that $\mathrm{H}_{\left(K^{(n)}\right)^{-}}-\left(\left\lceil\frac{n}{\sigma_{1}}\right\rceil\right) \geq m-2$. Let $\ell$ be the linear form $T_{1, \sigma_{1}+1}-T_{2,1}$ of $A^{\sim}$, and let ${ }^{-}$denote images in the ring $\overline{A^{\sim}}=A^{\sim} /(\ell)$. Clearly $\ell$ is a $A^{\sim}$ regular element. Recall that $T_{1, \sigma_{1}+1}$ is regular on $A^{\sim} /\left(K^{(n)}\right)^{\sim}$, see [22, the proof of 1.4]. Furthermore $T_{2,1}$ is an element of $K$ and hence its image in $A^{-} /\left(K^{(n)}\right)^{2}$ is nilpotent. Therefore $\ell=T_{1, \sigma_{1}+1}-T_{2,1}$ is regular on $A^{\sim} /\left(K^{(n)}\right)^{-}$. It follows that $\mathrm{H}_{\overline{\left(K^{(n)}\right)^{\sim}}}\left(\left\lceil\frac{n}{\sigma_{1}}\right\rceil\right)=\Delta \mathrm{H}_{\left(K^{(n)}\right)^{\sim}}\left(\left\lceil\frac{n}{\sigma_{1}}\right\rceil\right)$. However, $\left(K^{(n)}\right)^{\sim}$ is concentrated in degrees at least $\left\lceil\frac{n}{\sigma_{1}}\right\rceil$ and therefore $\Delta \mathrm{H}_{\left(K^{(n)}\right)^{-}}\left(\left\lceil\frac{n}{\sigma_{1}}\right\rceil\right)=\mathrm{H}_{\left(K^{(n)}\right)^{-}}\left(\left\lceil\frac{n}{\sigma_{1}}\right\rceil\right)$. On the other hand, $r\left(\overline{A^{\sim}} / \overline{\left.\left(K^{(n)}\right)^{\sim}\right)} \geq r\left(A^{\sim} /\left(K^{(n)}\right)^{\sim}\right)\right.$. Hence, it suffices to prove that if $\mathrm{H} \overline{\left(K^{(n)}\right)^{-}}\left(\left\lceil\frac{n}{\sigma_{1}}\right\rceil\right) \geq m-2$, then $r\left(\overline{A^{\sim}} / \overline{\left(K^{(n)}\right)^{\sim}}\right)<\left\lceil\frac{n}{\sigma_{1}}\right\rceil$. For this we wish to apply Theorem 4.5 to the integer $\left\lceil\frac{n}{\sigma_{1}}\right\rceil$ and the ideal $\overline{\left(K^{(n)}\right)^{`}}$ of the ring $\overline{A^{\vee}}$. Notice that $\overline{A^{-}}$is the homogeneous coordinate ring of a rational normal curve. In particular, it is a two dimensional Cohen-Macaulay domain with minimal multiplicity $m-2$. By Proposition 4.7 the ring $\overline{A^{`}}$ satisfies condition (4.6). Furthermore, $\overline{\left(K^{(n)}\right)^{`}}$ is a homogeneous ideal with $\operatorname{dim} \overline{A^{`}} / \overline{\left(K^{(n)}\right)^{`}}=1$; thus, Theorem 4.5 implies that $r\left(\overline{A^{`}} \overline{\left(K^{(n)}\right)^{`}}\right)<\left\lceil\frac{n}{\sigma_{1}}\right\rceil$ if $\Delta \mathrm{H}_{\overline{\left(K^{(n)}\right)^{-}}}\left(\left\lceil\frac{n}{\sigma_{1}}\right\rceil\right) \geq m-2$. But again, $\Delta \mathrm{H}_{\overline{\left(K^{(n)}\right)^{\nu}}}\left(\left\lceil\frac{n}{\sigma_{1}}\right\rceil\right)=\mathrm{H}_{\overline{\left(K^{(n)}\right)^{\nu}}}\left(\left\lceil\frac{n}{\sigma_{1}}\right\rceil\right)$. This completes the proof of the first implication.

Conversely, assume that $r\left(A^{\sim} /\left(K^{(n)}\right)^{\sim}\right)<\left\lceil\frac{n}{\sigma_{1}}\right\rceil$. Now let $\overline{A^{\smile}}$ denote the ring obtained from $A^{\sim}$ by a purely transcendental extension of the field $k$ and by factoring

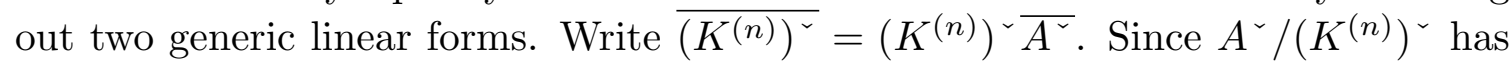
dimension two it follows that $r\left(\overline{A^{\sim}} / \overline{\left(K^{(n)}\right)^{\sim}}\right)=r\left(A^{\sim} /\left(K^{(n)}\right)^{\sim}\right)$, see [26]. Therefore $r\left(\overline{A^{\sim}} / \overline{\left.\left(K^{(n)}\right)^{\sim}\right)}<\left\lceil\frac{n}{\sigma_{1}}\right\rceil\right.$. Because $\overline{A^{\sim}} / \overline{\left(K^{(n)}\right)^{\sim}}$ is Artinian and $\overline{A^{\sim}}$ is a onedimensional standard graded Cohen-Macaulay ring with minimal multiplicity $m-2$, we conclude that $\mathrm{H}_{\overline{\left(K^{(n)}\right)^{v}}}\left(\left\lceil\frac{n}{\sigma_{1}}\right\rceil\right)=\mathrm{H}_{\overline{A^{v}}}\left(\left\lceil\frac{n}{\sigma_{1}}\right\rceil\right)=m-2$. Clearly,

$$
\mathrm{H}_{\left(K^{(n)}\right)^{\sim}}\left(\left\lceil\frac{n}{\sigma_{1}}\right\rceil\right) \geq \mathrm{H}_{\overline{\left(K^{(n)}\right)^{-}}}\left(\left\lceil\frac{n}{\sigma_{1}}\right\rceil\right) .
$$

Hence indeed $\mathrm{H}_{\left(K^{(n)}\right)^{\sim}}\left(\left\lceil\frac{n}{\sigma_{1}}\right\rceil\right) \geq m-2$. 


\section{Eagon-Northcott modules.}

Let $I$ be the ideal of (1.1). In Theorem 6.1 we record the graded betti numbers in the minimal homogeneous resolution of $I^{s}$ for all $s$. The main step in the proof of this Theorem is the calculation of $\lambda\left(I_{\mathfrak{z}}^{s}\right)$ for all positive integers $s$ and $\mathfrak{z}$ and we do this by calculating $\lambda\left((S / H)_{(u, s)}\right)$ and $\lambda\left(K_{(u, s)}^{(n)}\right)$ for each bi-degree $(u, s)$. The $S$-module $S / H$ is resolved by an Eagon-Northcott complex and we have identified a filtration $\left\{\mathcal{E}_{\boldsymbol{a}}\right\}$ of $K^{(n)}$ so that each factor $\mathcal{E}_{\boldsymbol{a}} / \mathcal{D}_{\boldsymbol{a}}$ is an "Eagon-Northcott module", in the sense that is resolved by an Eagon-Northcott complex. See [2, Section $2 \mathrm{C}]$ or [11, Section A2.6] for more information about these modules and complexes. We define the Eagon-Northcott modules in Definition 5.1. The Hilbert function of each Eagon-Northcott module, in the standard graded case, is given in Proposition 5.2. Lemma 5.4 and Corollary 5.5 show how to compute the Hilbert function of an Eagon-Northcott module in a bi-graded situation. The main result of the present section is Proposition 5.6, where we record the formula for $\lambda\left((S / H)_{(u, s)}\right)$ and $\lambda\left(\left(\mathcal{E}_{\boldsymbol{a}} / \mathcal{D}_{\boldsymbol{a}}\right)_{(u, s)}\right)$ for each eligible tuple $\boldsymbol{a}$ and each bi-degree $(u, s)$.

D10.5 Definition 5.1. Let $P$ be a ring, $E$ and $F$ be free $P$-modules of rank 2 and $c$, respectively, and $\Psi: F \rightarrow E$ be a homomorphism of $P$-modules. Define the EagonNorthcott module EN $[\Psi, P, r]$ by

$$
\operatorname{EN}[\Psi, P, r]= \begin{cases}\operatorname{coker}\left(E^{*} \otimes \bigwedge^{2} F \rightarrow F\right) & \text { if } r=-1 \\ P / I_{2}(\Psi) & \text { if } r=0 \\ \operatorname{Sym}_{r}(\operatorname{coker} \Psi) & \text { if } 1 \leq r .\end{cases}
$$

The defining map for $\operatorname{EN}[\Psi, P,-1]$ sends $u \otimes v$ to $\left[\Psi^{*}(u)\right](v)$. When there is no ambiguity about the ring $P$, we surpress the $P$ and write $\mathrm{EN}[\Psi, r]$ in place of $\operatorname{EN}[\Psi, P, r]$.

Convention. We define the binomial coefficient $\left(\begin{array}{l}j \\ i\end{array}\right)$ for all integers $i$ and $j$ by

$$
\left(\begin{array}{c}
j \\
i
\end{array}\right)=\left\{\begin{array}{cl}
\frac{j(j-1) \cdots(j-i+1)}{i !} & \text { if } 0<i, \\
1 & \text { if } 0=i, \text { and } \\
0 & \text { if } i<0 .
\end{array}\right.
$$

If $i$ and $j$ are integers with $0 \leq j$, then $\left(\begin{array}{l}j \\ i\end{array}\right)=\left(\begin{array}{c}j \\ j-i\end{array}\right)$. If $i$ is a nonnegative integer, then $\left(\begin{array}{c}-1 \\ i\end{array}\right)=(-1)^{i}$. 
P11.4 Proposition 5.2. Let $P$ be a standard graded polynomial ring over a field and let $\psi$ be a $2 \times$ c matrix of one-forms from $P$. Assume that ht $I_{2}(\psi)=c-1$ and let $D$ be the Krull dimension of $P / I_{2}(\psi)$. If $r$ and $s$ are integers, with $-1 \leq r \leq c-1$, then

ell

$$
\lambda\left(\operatorname{EN}[\psi, r]_{s}\right)=(r+1)\left(\begin{array}{c}
s+D-2 \\
s
\end{array}\right)+c\left(\begin{array}{c}
s+D-2 \\
s-1
\end{array}\right) .
$$

Remarks.

1. Notice that both sides of (5.3) are zero when $s<0$.

2. If $D=0$, then the right side of (5.3) is equal to

$$
\begin{cases}r+1, & \text { if } s=0, \\ c-(r+1), & \text { if } s=1, \text { and } \\ 0, & 2 \leq s\end{cases}
$$

3. If $D=1$, then the right side of (5.3) is equal to

$$
\begin{cases}r+1, & \text { if } s=0, \text { and } \\ c, & \text { if } 1 \leq s\end{cases}
$$

Proof. The proof is by induction on $D$. Start with $D=0$. In this case, the number of variables in $P$ is equal to ht $I_{2}(\psi)=c-1$. In particular, $\lambda\left(P_{1}\right)=c-1$. Let $F=P(-1)^{c}$ and $E=P^{2}$. We view $\psi$ as a map $\psi: F \rightarrow E$. First, fix $r \geq 1$. In this case, $\mathrm{EN}[\psi, r]$ is minimally presented by

$$
S_{r-1} E \otimes F \rightarrow S_{r} E \rightarrow \mathrm{EN}[\psi, r] \rightarrow 0,
$$

which is the same as

$$
P(-1)^{r c} \rightarrow P^{r+1} \rightarrow \mathrm{EN}[\psi, r] \rightarrow 0 .
$$

It is clear that $\lambda\left(\operatorname{EN}[\psi, r]_{0}\right)=r+1$. One may read that

$$
\lambda\left(\operatorname{EN}[\psi, r]_{1}\right)=(r+1) \lambda\left(P_{1}\right)-r c \lambda\left(P_{0}\right)=c-1-r .
$$

We know that $I_{2}(\psi)$ kills $\operatorname{EN}[\psi, r]$. However, $I_{2}(\psi)$ is equal to the square of the maximal ideal of $P$ (notice that $I_{2}(\psi) \subseteq \mathfrak{m}^{2}$ and both ideals of $P$ are minimally generated by $\left(\begin{array}{l}c \\ 2\end{array}\right)$ elements of $P_{2}$ ), and $\mathrm{EN}[\psi, r]$ is generated in degree zero; so $\mathrm{EN}[\psi, r]_{s}=0$ for all $s \geq 2$. 
It is very easy to see that the assertion is correct for $r=0$. We now consider $r=-1$. The module $\mathrm{EN}[\psi, r]$ is minimally presented by

$$
E^{*} \otimes \bigwedge^{2} F \rightarrow F \rightarrow \mathrm{EN}[\psi, r] \rightarrow 0,
$$

which is the same as

$$
P(-2)^{2\left(\begin{array}{c}
c \\
2
\end{array}\right)} \rightarrow P(-1)^{c} \rightarrow \mathrm{EN}[\psi, r] \rightarrow 0 .
$$

We can now read that

$$
\lambda\left(\operatorname{EN}[\psi, r]_{s}\right)= \begin{cases}0, & \text { if } s=0, \\
c, & \text { if } s=1, \text { and } \\
c \lambda\left(P_{1}\right)-2\left(\begin{array}{c}
c \\
2
\end{array}\right)=0, & \text { if } s=2 .\end{cases}
$$

Once again, all of the generators of $\operatorname{EN}(\psi, r)$ have the same degree. As soon as we know that $\mathrm{EN}[\psi, r]_{2}=0$, then we know that $\mathrm{EN}[\psi, r]_{s}=0$ for all $s \geq 2$.

Now we treat positive $D$. Suppose that the element $x$ of $P_{1}$ is regular on $P / I_{2}(\psi)$. Let $\bar{P}$ be $P /(x)$ and $\bar{\psi}$ be $\psi \otimes_{P} \bar{P}$. The module $\operatorname{EN}[r, \psi]$ is perfect (in the sense of [1, Def. 1.4.14]) and has the same associated prime ideals as $P / I_{2}(\psi)$. It follows that

$$
0 \rightarrow \mathrm{EN}[\psi, r](-1) \stackrel{x}{\rightarrow} \mathrm{EN}[\psi, r] \rightarrow \operatorname{EN}[\bar{\psi}, r] \rightarrow 0
$$

is an exact sequence; and therefore $\lambda\left(\operatorname{EN}[\psi, r]_{s}\right)=\sum_{i=0}^{s} \lambda\left(\operatorname{EN}[\bar{\psi}, r]_{i}\right)$.

We now study the Hilbert function of the Eagon-Northcott modules in a bigraded situation. The main algebraic tool is Lemma 5.4, which has nothing to do with grading. In Corollary 5.5, we apply Lemma 5.4 to the bigraded case of interest.

L10.11 Lemma 5.4. Adopt the notation of Definition 5.1. Assume that $F=F^{\prime} \oplus F^{\prime \prime}$ for free modules $F^{\prime}$ and $F^{\prime \prime}$ where $F^{\prime \prime}$ has rank 1 . Let $\Psi^{\prime}: F^{\prime} \rightarrow E$ be the restriction of $\Psi$ to $F^{\prime}$ and $\Psi^{\prime \prime}: F^{\prime \prime} \rightarrow E$ be the restriction of $\Psi$ to $F^{\prime \prime}$. Assume that

$$
\text { grade } I_{2}(\Psi) \geq c-1 \text {. }
$$

If $0 \leq r \leq c-1$, then there is a short exact sequence

$$
0 \rightarrow \mathrm{EN}\left[\Psi^{\prime}, r-1\right] \otimes F^{\prime \prime} \stackrel{\iota}{\rightarrow} \mathrm{EN}\left[\Psi^{\prime}, r\right] \stackrel{\pi}{\rightarrow} \mathrm{EN}[\Psi, r] \rightarrow 0,
$$

where $\pi$ is the natural surjection and

$$
\iota(m \otimes v)= \begin{cases}m \cdot \Psi^{\prime \prime}(v) & \text { for } 1 \leq r \\ \bigwedge^{2} \Psi(m \wedge v) & \text { for } r=0 .\end{cases}
$$


Proof. Recall that the Eagon-Northcott complex that is associated to $\operatorname{EN}[\Psi, r]$ is $\mathfrak{E N}[\Psi, r] \bullet$ with

$$
\mathfrak{E N}[\Psi, r]_{p}= \begin{cases}\operatorname{Sym}_{r-p} E \otimes \bigwedge^{p} F & \text { if } 0 \leq p \leq r \\ D_{p-r} E^{*} \otimes \bigwedge^{p+1} F & \text { if } r+1 \leq p .\end{cases}
$$

Recall also, that if grade $I_{2}(\Psi) \geq c-1$, then $\mathfrak{E N}[\Psi, r] \bullet$ is a resolution of $\operatorname{EN}[\Psi, r]$. In the present situation, the decomposition $F=F^{\prime} \oplus F^{\prime \prime}$ induces a short exact sequence of modules

$$
0 \rightarrow \bigwedge^{p} F^{\prime} \rightarrow \bigwedge^{p} F \rightarrow \bigwedge^{p-1} F^{\prime} \otimes F^{\prime \prime} \rightarrow 0
$$

for all $p$. Furthermore, these short exact sequences of modules induce a short exact sequence of complexes

$$
0 \rightarrow \mathfrak{E N}\left[\Psi^{\prime}, r\right] \bullet \rightarrow \mathfrak{E N}[\Psi, r] \bullet \rightarrow \mathfrak{E N}\left[\Psi^{\prime}, r-1\right] \bullet[-1] \otimes F^{\prime \prime} \rightarrow 0,
$$

for all $r$. The corresponding long exact sequence of homology includes

$$
\mathrm{H}_{1}\left(\mathfrak{E N}[\Psi, r]_{\bullet}\right) \rightarrow \mathrm{EN}\left[\Psi^{\prime}, r-1\right] \otimes F^{\prime \prime} \stackrel{\iota}{\rightarrow} \mathrm{EN}\left[\Psi^{\prime}, r\right] \stackrel{\pi}{\longrightarrow} \mathrm{EN}[\Psi, r] \rightarrow 0 .
$$

The hypothesis grade $I_{2}(\Psi) \geq c-1$ ensures that $\mathrm{H}_{1}\left(\mathfrak{E N}[\Psi, r]_{\bullet}\right)=0$.

L10.11' Corollary 5.5. Retain the hypotheses of Lemma 5.4. Suppose that the ring $P$ is equal to $P^{\prime}[x, y]$ where $P^{\prime}$ is a standard graded polynomial ring over the field $k$ and $x$ and $y$ are new variables. View $P$ as a bi-graded ring. The variables $x$ and $y$ have degree $(1,0)$. Each variable from $P^{\prime}$ has degree $(0,1)$. Suppose $\Psi^{\prime}$ is a $(c-1) \times 2$ matrix of linear forms from $P^{\prime}$ and $\Psi^{\prime \prime}=\left[\begin{array}{l}y \\ x\end{array}\right]$. Let $R$ be the standard graded polynomial ring $k[x, y]$. If $(u, s)$ is any bi-degree and $r$ is any integer with $0 \leq r \leq c$, then

$$
\lambda\left(\operatorname{EN}[\Psi, P, r]_{(u, s)}\right)=\lambda\left(R_{u}\right) \lambda\left(\operatorname{EN}\left[\Psi^{\prime}, P^{\prime}, r\right]_{s}\right)-\lambda\left(R(-1)_{u}\right) \lambda\left(\operatorname{EN}\left[\Psi^{\prime}, P^{\prime}, r-1\right]_{s}\right) .
$$

Proof. Apply Lemma 5.4 to obtain the short exact sequence

$$
0 \rightarrow \mathrm{EN}\left[\Psi^{\prime}, P, r-1\right](-1,0) \rightarrow \mathrm{EN}\left[\Psi^{\prime}, P, r\right] \rightarrow \mathrm{EN}[\Psi, P, r] \rightarrow 0 .
$$

We have $P=R \otimes_{k} P^{\prime}$. The map $\Psi^{\prime}: P(-1)^{m-2} \rightarrow P^{2}$ is the same as

$$
1 \otimes \Psi^{\prime}: R \otimes_{k} P^{\prime}(-1)^{m-2} \rightarrow R \otimes_{k}{P^{\prime 2}}^{2}
$$


and therefore, $\mathrm{EN}\left[\Psi^{\prime}, P, r\right]=R \otimes_{k} \operatorname{EN}\left[\Psi^{\prime}, P^{\prime}, r\right]$. It follows that

$$
\begin{aligned}
& \lambda\left((\operatorname{EN}[\Psi, P, r])_{(u, s)}\right) \\
& =\lambda\left(\left(R \otimes_{k} \operatorname{EN}\left[\Psi^{\prime}, P^{\prime}, r\right]\right)_{(u, s)}\right)-\lambda\left(\left(R(-1) \otimes_{k} \operatorname{EN}\left[\Psi^{\prime}, P^{\prime}, r-1\right]\right)_{(u, s)}\right) \\
& =\lambda\left(R_{u}\right) \lambda\left(\operatorname{EN}\left[\Psi^{\prime}, P^{\prime}, r\right]_{s}\right)-\lambda\left(R(-1)_{u}\right) \lambda\left(\operatorname{EN}\left[\Psi^{\prime}, P^{\prime}, r-1\right]_{s}\right) .
\end{aligned}
$$

The rest of this section is devoted to proving Proposition 5.6. Adopt the notation of Definition 1.5 with (3.1). Recall the notion of eligible $k$-tuple $\boldsymbol{a}=\left(a_{1}, \ldots, a_{k}\right)$, as well as $f(\boldsymbol{a})$ and $r(\boldsymbol{a})$, from the statement of Theorem 3.2. In [22] we put a total order on the set of eligible tuples. For eligible tuples $\boldsymbol{b}>\boldsymbol{a}$ we define ideals $\mathcal{E}_{\boldsymbol{b}} \subseteq \mathcal{E}_{\boldsymbol{a}}$ of $A$ by induction. There is no convenient way to denote the eligible tuple which is immediately larger than a particular eligible tuple $\boldsymbol{a}$; consequently, we define two parallel collections of ideals $\left\{\mathcal{E}_{\boldsymbol{a}}\right\}$ and $\left\{\mathcal{D}_{\boldsymbol{a}}\right\}$ simultaneously. The ideal $\mathcal{D}_{\emptyset}$ is equal to zero. If $\boldsymbol{a}$ is an eligible tuple of positive length, then $\mathcal{D}_{\boldsymbol{a}}=\sum_{\boldsymbol{b}>\boldsymbol{a}} \mathcal{E}_{\boldsymbol{b}}$. If $\boldsymbol{a}$ is an arbitrary eligible tuple, then

$$
\mathcal{E}_{\boldsymbol{a}}=\mathcal{D}_{\boldsymbol{a}}+T^{\boldsymbol{a}} T_{k+1,1}^{f(\boldsymbol{a})}\left(T_{k+1,1}, \ldots, T_{k+1, r(\boldsymbol{a})}\right) .
$$

We have a filtration of $K^{(n)}$ :

$$
(0) \subsetneq \mathcal{E}_{\emptyset} \subsetneq \cdots \subsetneq \mathcal{E}_{0^{\ell-1}}=K^{(n)},
$$

where $0^{s}$ is the $s$-tuple $(0, \ldots, 0)$. It is also shown in [22] that the factor module $\mathcal{E}_{\boldsymbol{a}} / \mathcal{D}_{\boldsymbol{a}}$ is isomorphic to the Eagon-Northcott module

$$
\operatorname{EN}\left[\psi_{>k}, S / P_{k}, r(\boldsymbol{a})-1\right]\left(-t_{\boldsymbol{a}}\right),
$$

where $\psi_{>k}$ is the submatrix $\left[\begin{array}{lll}\psi_{k+1} & \cdots & \psi_{\ell}\end{array}\right]$ of $\psi, P_{k}$ is the ideal

$$
P_{k}=k\left[\left\{T_{i, j} \mid 1 \leq i \leq k, 1 \leq j \leq \sigma_{i}\right\}\right]
$$

of $S$, and $t_{\boldsymbol{a}}$ is the twist

$$
t_{\boldsymbol{a}}= \begin{cases}\left(0, \sum_{u=1}^{k} a_{u}+f(\boldsymbol{a})+1\right), & \text { if } k<\rho, \\ \left(f(\boldsymbol{a})+1, \sum_{u=1}^{k} a_{u}\right), & \text { if } k=\rho .\end{cases}
$$


5main Proposition 5.6. Adopt the notation of Definition 1.5 with (3.1). Let $(u, s)$ be an arbitrary bi-degree.

(a)

$\lambda\left((S / H)_{(u, s)}\right)=\lambda\left(R_{u}\right)\left(\left(\begin{array}{c}s+1 \\ s\end{array}\right)+(m-2)\left(\begin{array}{c}s+1 \\ s-1\end{array}\right)\right)-\lambda\left(R(-1)_{u}\right)(m-2)\left(\begin{array}{c}s+1 \\ s-1\end{array}\right)$

(b)

$$
\lambda\left(\left(\mathcal{E}_{\emptyset} / \mathcal{D}_{\emptyset}\right)_{(u, s)}\right)=\left\{\begin{array}{l}
\lambda\left(R_{u}\right)\left[r(\emptyset)\left(\begin{array}{c}
s-f(\emptyset) \\
s-f(\emptyset)-1
\end{array}\right)+(m-2)\left(\begin{array}{c}
s-f(\emptyset) \\
s-f(\emptyset)-2
\end{array}\right)\right] \\
-\lambda\left(R(-1)_{u}\right)\left[(r(\emptyset)-1)\left(\begin{array}{c}
s-f(\emptyset) \\
s-f(\emptyset)-1
\end{array}\right)+(m-2)\left(\begin{array}{c}
s-f(\emptyset) \\
s-f(\emptyset)-2
\end{array}\right)\right]
\end{array}\right.
$$

(c) If $\boldsymbol{a}=\left(a_{1}\right)$ is an eligible 1-tuple, then $\lambda\left(\left(\mathcal{E}_{\boldsymbol{a}} / \mathcal{D}_{\boldsymbol{a}}\right)_{(u, s)}\right)$ is equal to

$$
\begin{cases}\chi\left(a_{1} \leq s\right) \lambda\left(R\left(a_{1} \sigma_{1}-n\right)_{u}\right), & \text { if } \rho=1, \text { or } \\
\chi\left(a_{1}+f\left(a_{1}\right)+1 \leq s\right)\left(\begin{array}{c}
\lambda\left(R_{u}\right)\left(a_{1} \sigma_{1}-n+1+\sigma_{2}\left(s-a_{1}\right)\right) \\
-\lambda\left(R(-1)_{u}\right)\left(a_{1} \sigma_{1}-n+\sigma_{2}\left(s-a_{1}\right)\right),
\end{array}\right) & \text { if } \rho=2 .\end{cases}
$$

(d) If $\boldsymbol{a}=\left(a_{1}, a_{2}\right)$ is an eligible 2-tuple then

$$
\lambda\left(\left(\mathcal{E}_{\boldsymbol{a}} / \mathcal{D}_{\boldsymbol{a}}\right)_{(u, s)}\right)=\chi\left(s=a_{1}+a_{2}\right) \lambda\left(R\left(a_{1} \sigma_{1}+a_{2} \sigma_{2}-n\right)_{u}\right) .
$$

Proof. For (a) and (b) we apply Corollary 5.5 with $P^{\prime}=k\left[T_{1}, \ldots, T_{m}\right]$ and $\Psi^{\prime}$ equal to the first $m-2$ columns of $\psi$. Thus,

$$
\begin{aligned}
\lambda\left((S / H)_{(u, s)}\right) & =\lambda\left((\operatorname{EN}[\psi, S, 0])_{(u, s)}\right) \\
& =\lambda\left(R_{u}\right) \lambda\left(\operatorname{EN}\left[\Psi^{\prime}, P^{\prime}, 0\right]_{s}\right)-\lambda\left(R(-1)_{u}\right) \lambda\left(\operatorname{EN}\left[\Psi^{\prime}, P^{\prime},-1\right]_{s}\right)
\end{aligned}
$$

and

$$
\begin{aligned}
\lambda\left(\left(\mathcal{E}_{\emptyset} / \mathcal{D}_{\emptyset}\right)_{(u, s)}\right) & =\lambda\left((\operatorname{EN}[\psi, S, r(\emptyset)-1](0,-f(\emptyset)-1))_{(u, s)}\right) \\
& =\left\{\begin{array}{c}
\lambda\left(R_{u}\right) \lambda\left(\left(\operatorname{EN}\left[\Psi^{\prime}, P^{\prime}, r(\emptyset)-1\right](-f(\emptyset)-1)\right)_{s}\right) \\
-\lambda\left(R(-1)_{u}\right) \lambda\left(\left(\operatorname{EN}\left[\Psi^{\prime}, P^{\prime}, r(\emptyset)-2\right](-f(\emptyset)-1)\right)_{s}\right) .
\end{array}\right.
\end{aligned}
$$

Apply Proposition 5.2, with $c=m-2$ and $D=3$, to establish (a) and (b). 
Take $\boldsymbol{a}=\left(a_{1}\right)$ to be an eligible 1-tuple with $\rho=1$. Apply Corollary 5.5 with $P^{\prime}=k\left[T_{m}\right]$ and $\Psi^{\prime}$ equal to the zero map. In this case, $r(\boldsymbol{a})=1, f(\boldsymbol{a})+1=n-a_{1} \sigma_{1}$, $\mathrm{EN}\left[0, P^{\prime}, 0\right]=P^{\prime}$, and $\operatorname{EN}\left[0, P^{\prime},-1\right]=0$. We have

$$
\begin{aligned}
\lambda\left(\left(\mathcal{E}_{\boldsymbol{a}} / \mathcal{D}_{\boldsymbol{a}}\right)_{(u, s)}\right) & =\lambda\left(\left(\operatorname{EN}\left[\left[\begin{array}{c}
y \\
x
\end{array}\right], P^{\prime}[x, y], 0\right]\left(a_{1} \sigma_{1}-n,-a_{1}\right)\right)_{(u, s)}\right) \\
& =\lambda\left(R\left(a_{1} \sigma_{1}-n\right)_{u}\right) \lambda\left(P^{\prime}\left(-a_{1}\right)_{s}\right) .
\end{aligned}
$$

If $\boldsymbol{a}=\left(a_{1}, a_{2}\right)$ is an eligible 2-tuple, then $\rho$ must equal 2, $r(\boldsymbol{a})=1, f(\boldsymbol{a})+1=$ $n-a_{1} \sigma_{1}-a_{2} \sigma_{2}$,

$$
\begin{aligned}
\lambda\left(\left(\mathcal{E}_{\boldsymbol{a}} / \mathcal{D}_{\boldsymbol{a}}\right)_{(u, s)}\right) & =\lambda\left(\left(\operatorname{EN}\left[\left[\begin{array}{l}
y \\
x
\end{array}\right], k[x, y], 0\right]\left(a_{1} \sigma_{1}+a_{2} \sigma_{2}-n,-a_{1}-a_{2}\right)\right)_{(u, s)}\right) \\
& =\lambda\left(R\left(a_{1} \sigma_{1}+a_{2} \sigma_{2}-n\right)_{u}\right) \lambda\left(k\left(-a_{1}-a_{2}\right)_{s}\right) .
\end{aligned}
$$

Finally, let $\boldsymbol{a}=\left(a_{1}\right)$ be an eligible 1-tuple with $\rho=2$. Apply Corollary 5.5 with $P^{\prime}=k\left[T_{2,1}, \ldots, T_{2, \sigma_{2}+1}\right]$ and $\Psi^{\prime}=\psi_{2}$ to see that

$$
\begin{aligned}
\lambda\left(\left(\mathcal{E}_{\boldsymbol{a}} / \mathcal{D}_{\boldsymbol{a}}\right)_{(u, s)}\right) & =\lambda\left(\left(\operatorname{EN~}\left[\left[\begin{array}{ll}
\psi_{2} & \psi_{3}
\end{array}\right], P^{\prime}[x, y], r(\boldsymbol{a})-1\right]\left(0,-a_{1}-f(\boldsymbol{a})-1\right)\right)_{(u, s)}\right) \\
& =\left\{\begin{array}{c}
\lambda\left(R_{u}\right) \lambda\left(\left(\operatorname{EN}\left[\psi_{2}, P^{\prime}, r(\boldsymbol{a})-1\right]\left(-a_{1}-f(\boldsymbol{a})-1\right)\right)_{s}\right) \\
-\lambda\left(R(-1)_{u}\right) \lambda\left(\left(\operatorname{EN}\left[\psi_{2}, P^{\prime}, r(\boldsymbol{a})-2\right]\left(-a_{1}-f(\boldsymbol{a})-1\right)\right)_{s}\right) .
\end{array}\right.
\end{aligned}
$$

Apply Proposition 5.2, with $c=\sigma_{2}$ and $D=2$, to complete the calculation.

\section{The resolution of $I^{s}$.}

We resolve every power of the ideal $I$ of Definition 1.5. Our answer is expressed in terms of the parameter " $a$ ", which is equal to the number of non-linear columns in the matrix which presents $I^{s}$. The resolution depends on the shape of the partition $\sigma$ which corresponds to $I$.

pwrs4 Theorem 6.1. Let I be the ideal of Definition 1.5 and $s$ be a positive integer. The minimal homogeneous resolution of $I^{s}$ has the form

$$
0 \rightarrow R(-s d-1)^{b} \oplus \mathbb{F} \rightarrow R(-s d)^{b_{0}} \rightarrow I^{s} \rightarrow 0,
$$

with $b_{0}=b+a+1$.

(1) If $\rho=1$, then $\mathbb{F}=\sum_{u=0}^{a-1} R\left(-s d+u \sigma_{1}-n\right), b=s d+\left(\begin{array}{l}a \\ 2\end{array}\right) \sigma_{1}-a n$, and

$$
a=\min \left\{s,\left\lceil\frac{n-1}{\sigma_{1}}\right\rceil\right\}
$$


(2) If $\rho=2$ and $\sigma_{1}>\sigma_{2}$, then $\mathbb{F}=\sum_{u=0}^{a-1} R\left(-s d+u\left(\sigma_{1}-\sigma_{2}\right)+(s-1) \sigma_{2}-n\right)$,

$$
\begin{aligned}
& b=s\left(d+a \sigma_{2}\right)+\left(\begin{array}{l}
a \\
2
\end{array}\right)\left(\sigma_{1}-\sigma_{2}\right)-a\left(n+\sigma_{2}\right), \text { and } \\
& a= \begin{cases}\min \left\{s,\left\lceil\frac{n-(s-1) \sigma_{2}-1}{\sigma_{1}-\sigma_{2}}\right\rceil\right\}, & \text { if } s \leq \frac{n-2}{\sigma_{2}}+1, \\
0, & \text { if } \frac{n-1}{\sigma_{2}}+1 \leq s .\end{cases}
\end{aligned}
$$

(3) If $\rho=2$ and $\sigma_{1}=\sigma_{2}$, then $\mathbb{F}=R\left(-s d+(s-1) \sigma_{2}-n\right)^{a}$,

$$
b=s\left(d+a \sigma_{2}\right)-a\left(n+\sigma_{2}\right) \quad \text { and } \quad a= \begin{cases}s, & \text { if } s \leq \frac{n-2}{\sigma_{2}}+1 \text { and } \\ 0, & \text { if } \frac{n-1}{\sigma_{2}}+1 \leq s .\end{cases}
$$

Remark. It is worth noting that the non-linear columns in the presenting matrix for $I^{s}$ all have the same degree for $\sigma_{1}=\sigma_{2}$; however, these columns have distinct degrees in other two cases.

Proof. The ring $S$ is bi-graded and the quotient map

$$
S \rightarrow S / \mathcal{A}=\mathcal{R}(I)
$$

sends $S_{(u, s)} \rightarrow R_{u} I^{s} t^{s}=I_{u+s d^{s}}^{s} t^{s}$, where $d$ is the degree of the generators of $I$; so,

$$
\lambda\left(I_{u+d s}^{s}\right)=\lambda\left((S / \mathcal{A})_{(u, s)}\right),
$$

and, for all integers $s$ and $\mathfrak{z}$,

$$
\lambda\left(I_{\mathfrak{z}}^{s}\right)=\lambda\left((S / \mathcal{A})_{(\mathfrak{z}-d s, s)}\right) .
$$

The short exact sequence

$$
0 \rightarrow \mathcal{A} / H \rightarrow S / H \rightarrow S / \mathcal{A} \rightarrow 0
$$

gives

$$
\lambda\left((S / \mathcal{A})_{z}\right)=\lambda\left((S / H)_{z}\right)-\lambda\left((\mathcal{A} / H)_{z}\right) .
$$

The element $g / y^{n}$ of the quotient field of $A=S / H$ has degree $(0,1)$, since $g$ has degree $(n, 1)$ and $y^{n}$ has degree $(n, 0)$, and the isomorphism $g / y^{n}: K^{(n)} \rightarrow \mathcal{A} A$ of ideals satisfies $\lambda\left(K_{(u, s-1)}^{(n)}\right)=\lambda\left((\mathcal{A} / H)_{(u, s)}\right)$. It follows that

$$
\lambda\left(I_{\mathfrak{z}}^{s}\right)=\lambda\left((S / H)_{(\mathfrak{z}-d s, s)}\right)-\lambda\left(K_{(\mathfrak{z}-d s, s-1)}^{(n)}\right) .
$$


We have identified a filtration

$$
\left\{\mathcal{E}_{\boldsymbol{a}} \mid \boldsymbol{a} \text { is an eligible tuple }\right\}
$$

of $K^{(n)} ;$ thus

$\operatorname{lam} 4 \quad(6.2)$

$$
\lambda\left(I_{\mathfrak{z}}^{s}\right)=\lambda\left((S / H)_{(\mathfrak{z}-d s, s)}\right)-\sum_{\boldsymbol{a}} \lambda\left(\left(\mathcal{E}_{\boldsymbol{a}} / \mathcal{D}_{\boldsymbol{a}}\right)_{(\mathfrak{z}-d s, s-1)}\right) .
$$

Each length on the right side of (6.2) has been calculated in Proposition 5.6. We have

$$
\lambda\left(I_{\mathfrak{z}}^{s}\right)=b_{0} \lambda\left(R(-s d)_{\mathfrak{z}}\right)-N_{1} \lambda\left(R(-s d-1)_{\mathfrak{z}}\right)-N_{2}
$$

for

$$
N_{2}= \begin{cases}\sum_{\left(a_{1}, a_{2}\right) \text { eligible }} \chi\left(a_{1}+a_{2}=s-1\right) \lambda\left(R\left(-s d+a_{1} \sigma_{1}+a_{2} \sigma_{2}-n\right)_{\mathfrak{z}}\right), & \text { if } \rho=2, \\ \sum_{\left(a_{1}\right) \text { eligible }} \chi\left(a_{1} \leq s-1\right) \lambda\left(R\left(-s d+a_{1} \sigma_{1}-n\right)_{\mathfrak{z}}\right), & \text { if } \rho=1,\end{cases}
$$

and integers $b_{0}$ and $N_{1}$. (There is no difficulty in recording the exact values of $b_{0}$ and $N_{1}$, but this is not necessary.)

When $\rho=2$, we simplify $N_{2}$ by replacing $a_{2}$ with $s-1-a_{1}$. The parameter $a_{1}$ must satisfy:

$$
0 \leq a_{1} \leq s-1 \quad \text { and } \quad a_{1} \sigma_{1}+\left(s-1-a_{1}\right) \sigma_{2}<n
$$

Thus,

form'

$$
N_{2}=N_{2}^{\prime} \lambda\left(R(-s d-1)_{\mathfrak{z}}\right)+N_{2}^{\prime \prime}
$$

for $N_{2}^{\prime}=\sum_{a_{1}=0}^{s-1} \chi\left(a_{1}\left(\sigma_{1}-\sigma_{2}\right)+(s-1) \sigma_{2}-n=-1\right)$ and

$$
N_{2}^{\prime \prime}=\sum_{a_{1}=0}^{s-1} \chi\left(a_{1}\left(\sigma_{1}-\sigma_{2}\right)+(s-1) \sigma_{2}-n \leq-2\right) \lambda\left(R\left(-s d+a_{1}\left(\sigma_{1}-\sigma_{2}\right)+(s-1) \sigma_{2}-n\right)_{\mathfrak{z}}\right) .
$$

When $\rho=1$, we write $N_{2}$ in the form (6.4) with $N_{2}^{\prime}=\sum_{a_{1}=0}^{s-1} \chi\left(a_{1} \sigma_{1}-n=-1\right)$ and

$$
N_{2}^{\prime \prime}=\sum_{a_{1}=0}^{s-1} \chi\left(a_{1} \sigma_{1}-n \leq-2\right) \lambda\left(R\left(-s d+a_{1} \sigma_{1}-n\right)_{\mathfrak{z}}\right) .
$$


Let $b=N_{1}+N_{2}^{\prime}$. Apply Lemma 6.6 to see that the minimal resolution of $I^{s}$ is

$$
0 \rightarrow R(-s d-1)^{b} \oplus \mathbb{F} \rightarrow R(-s d)^{b_{0}} \rightarrow I^{s} \rightarrow 0,
$$

for $\mathbb{F}$ equal to

$$
\sum_{a_{1}=0}^{s-1} \chi\left(a_{1}\left(\sigma_{1}-\sigma_{2}\right)+(s-1) \sigma_{2}-n \leq-2\right) R\left(-s d+a_{1}\left(\sigma_{1}-\sigma_{2}\right)+(s-1) \sigma_{2}-n\right),
$$

if $\rho=2$; or

$$
\sum_{a_{1}=0}^{s-1} \chi\left(a_{1} \sigma_{1}-n \leq-2\right) R\left(-s d+a_{1} \sigma_{1}-n\right),
$$

if $\rho=1$. Notice that the rank of $\mathbb{F}$ is equal to the number of non-linear columns in the presenting matrix for $I^{s}$. We next express $\mathbb{F}$ in a more transparent manner.

When $\rho=1$, the constraint $a_{1} \sigma_{1}-n \leq-2$ is equivalent to

$$
a_{1} \leq\left\lfloor\frac{n-2}{\sigma_{1}}\right\rfloor=\left\lceil\frac{n-1}{\sigma_{1}}\right\rceil-1
$$

and

$$
\mathbb{F}=\sum_{a_{1}=0}^{a-1} R\left(-s d+a_{1} \sigma_{1}-n\right),
$$

for $a=\min \left\{s,\left\lceil\frac{n-1}{\sigma_{1}}\right\rceil\right\}$.

Take $\rho=2$. The parameter $a_{1}$ is non-negative; so, $\mathbb{F}$ is zero if $\frac{n-1}{\sigma_{2}}+1 \leq s$. We think about $s \leq \frac{n-2}{\sigma_{2}}+1$. If $\sigma_{2}=\sigma_{1}$, then $\chi\left((s-1) \sigma_{2}-n \leq-2\right)=1$ and

$$
\mathbb{F}=\sum_{a_{1}=0}^{s-1} R\left(-s d+(s-1) \sigma_{2}-n\right)=R\left(-s d+(s-1) \sigma_{2}-n\right)^{s} .
$$

If $\sigma_{1}>\sigma_{2}$, then

$$
\begin{aligned}
& a_{1}\left(\sigma_{1}-\sigma_{2}\right)+(s-1) \sigma_{2}-n \leq-2 \Longleftrightarrow \\
& \quad a_{1} \leq\left\lfloor\frac{n-(s-1) \sigma_{2}-2}{\sigma_{1}-\sigma_{2}}\right\rfloor=\left\lceil\frac{n-(s-1) \sigma_{2}-1}{\sigma_{1}-\sigma_{2}}\right\rceil-1,
\end{aligned}
$$

and $\mathbb{F}=\sum_{a_{1}=0}^{a-1} R\left(-s d+a_{1}\left(\sigma_{1}-\sigma_{2}\right)+(s-1) \sigma_{2}-n\right)$, for

$$
a= \begin{cases}\min \left\{s,\left\lceil\frac{n-(s-1) \sigma_{2}-1}{\sigma_{1}-\sigma_{2}}\right\rceil\right\}, & s \leq \frac{n-2}{\sigma_{2}}+1, \\ 0, & \text { if } \rho=2, \frac{n-1}{\sigma_{2}}+1 \leq s\end{cases}
$$


Finally, we see that the values of $b_{0}$ and $b$ are completely determined by $a$. Indeed, rank is additive on short exact sequences; so, $b_{0}=b+a+1$. Also, $I^{s}$ is generated by the maximal minors of the matrix which presents $I^{s}$. In other words, $s d$ is equal to the sum of the column degrees of this presenting matrix; that is,

$\operatorname{tag} 4$

$$
\begin{aligned}
b & =s d+\sum_{a_{1}=0}^{a-1}\left(a_{1}\left(\sigma_{1}-\sigma_{2}\right)+(s-1) \sigma_{2}-n\right) \\
& =s d+\left(\begin{array}{l}
a \\
2
\end{array}\right)\left(\sigma_{1}-\sigma_{2}\right)+(s-1) \sigma_{2} a-n a .
\end{aligned}
$$

L19.14 Lemma 6.6. Let $M$ be a homogeneous module of projective dimension one over the standard graded polynomial ring $R$. Suppose that all of the generators of $M$ have degree D. Suppose further that $b_{0}, b_{1}$ and $t_{1} \leq t_{2} \leq \cdots \leq t_{b_{1}}$ are integers which satisfy $D<t_{1}$ and

$$
\lambda\left(M_{\mathfrak{z}}\right)=b_{0} \lambda\left(R(-D)_{\mathfrak{z}}\right)-\sum_{i=1}^{b_{1}} \lambda\left(R\left(-t_{i}\right)_{\mathfrak{z}}\right)
$$

for all integers $\mathfrak{z}$. Then the minimal homogeneous resolution of $M$ has the form

$$
0 \rightarrow \bigoplus_{i=1}^{b_{1}} R\left(-t_{i}\right) \rightarrow R(-D)^{b_{0}} \rightarrow M \rightarrow 0 .
$$

Proof. The hypotheses ensure that the minimal homogeneous resolution of $M$ has the form

$$
0 \rightarrow \bigoplus_{i=1}^{b_{1}^{\prime}} R\left(-t_{i}^{\prime}\right) \rightarrow R(-D)^{b_{0}^{\prime}} \rightarrow M \rightarrow 0
$$

for some integers $b_{0}^{\prime}, b_{1}^{\prime}$, and $t_{1}^{\prime} \leq t_{2}^{\prime} \leq \cdots \leq t_{b_{1}^{\prime}}^{\prime}$ with $D<t_{1}^{\prime}$. Use (6.7) to compute the Hilbert function of $M$; so

$$
b_{0} \lambda\left(R(-D)_{\mathfrak{z}}\right)-\sum_{i=1}^{b_{1}} \lambda\left(R\left(-t_{i}\right)_{\mathfrak{z}}\right)=b_{0}^{\prime} \lambda\left(R(-D)_{\mathfrak{z}}\right)-\sum_{i=1}^{b_{1}^{\prime}} \lambda\left(R\left(-t_{i}^{\prime}\right)_{\mathfrak{z}}\right),
$$

for all integers $\mathfrak{z}$. It follows that the free modules

$$
\mathbb{F}=R(-D)^{b_{0}} \oplus \bigoplus_{i=1}^{b_{1}^{\prime}} R\left(-t_{i}^{\prime}\right) \quad \text { and } \quad \mathbb{F}^{\prime}=R(-D)^{b_{0}^{\prime}} \oplus \bigoplus_{i=1}^{b_{1}} R\left(-t_{i}\right)
$$

have the same Hilbert function. This forces the free $R$-modules $\mathbb{F}$ and $\mathbb{F}^{\prime}$ to be equal; in other words, they have the exact same twists: $b_{0}=b_{0}^{\prime}, b_{1}=b_{1}^{\prime}$, and $t_{i}=t_{i}^{\prime}$ for all $i$.

The first two assertions of the following result may be read from the resolution of Theorem 6.1. A different proof of these results may be found in Corollary 2.12. 
reg Corollary 6.8. Let $I$ be the ideal of Definition 1.5 and $s$ be a positive integer.

(1) If $\rho=1$, then $\operatorname{reg} I^{s}=s d+n-1$ for all $s \geq 1$.

(2) If $\rho=2$, then $\operatorname{reg} I^{s}=s d$ if and only if $\frac{n-1}{\sigma_{2}}+1 \leq s$.

(3) The following statements are equivalent:

(a) $I^{s}=(x, y)^{s d}$,

(b) the minimal homogeneous resolution of $I^{s}$ has the form

$$
0 \rightarrow R(-s d-1)^{b-1} \rightarrow R(-s d)^{b} \rightarrow I^{s} \rightarrow 0,
$$

for some $b$,

(c) $\rho=2$ and $\frac{n-1}{\sigma_{2}}+1 \leq s$, or $\rho=1$ and $n=1$, and

(d) $a=0$.

(e) $\operatorname{reg} I^{s}=s d$.

Proof. We prove (3). The trick (6.5) shows that (a) and (b) are equivalent. The parameter $a$ is equal to the number of non-linear columns in the presenting matrix for $I^{s}$, so (d) and (b) are equivalent. The equivalence of (d) and (c) may be read from Theorem 6.1. Assertions (1) and (2) show that (c) and (e) are equivalent.

Let $B$ be a standard graded algebra over a field and let $q_{B}(s)$ be the Hilbert polynomial of $B$. It follows that $q_{B}(s)=\lambda\left(B_{s}\right)$ for all large $s$. The postulation number of $B$ is

$$
p(B)=\max \left\{s \mid q_{B}(s) \neq \lambda\left(B_{s}\right)\right\} .
$$

last Corollary 6.9. If $I$ is given in Definition 1.5, then

$$
p(\mathcal{F}(I))= \begin{cases}\left\lceil\frac{n-1}{\sigma_{2}}\right\rceil, & \text { if } \rho=2, \\ \left\lceil\frac{n-1}{\sigma_{1}}\right\rceil-1, & \text { if } \rho=1 .\end{cases}
$$

Proof. The Hilbert function and the Hilbert polynomial of $\mathcal{F}(I)$ may be read from Theorem 6.1: $\mathrm{H}_{\mathcal{F}(I)}(s)$ is equal to " $b_{0}$ ", written as a function of $s$ and

$$
q_{\mathcal{F}(I)}(s)= \begin{cases}s d+\left(\begin{array}{c}
a \\
2
\end{array}\right) \sigma_{1}-a n+a+1, & \text { if } \rho=1, \\
s d+1, & \text { if } \rho=2,\end{cases}
$$

for $a=\left\lceil\frac{n-1}{\sigma_{1}}\right\rceil$. The calculation of $p(\mathcal{F}(I))$ when $\rho=2$ is explicitly given in Corollary 6.8. A similar calculation is used when $\rho=1$.

Acknowledgment. This work was conducted while the first author was on sabbatical. He appreciates the hospitality he received at Purdue University and the University of Notre Dame. Also, we appreciate that Wolmer Vasconcelos made us aware of Conjecture 4.5 in [16]. This conjecture, later established by [9], is the starting point of this project. 


\section{REFERENCES}

$\mathrm{BH}$

BV88

B01

B07

BC05

BJ03

C06

C08

CHW

CHT

E95

$\mathrm{EH}$

EM04

$\mathrm{H}$

Но

HSV

HM

HU

J12

J11

Ko
1. W. Bruns and J. Herzog, Cohen-Macaulay rings, Cambridge studies in advanced mathematics 39, Cambridge University Press, Cambridge, 1993.

2. W. Bruns and U. Vetter, Determinantal rings, Lecture Notes in Mathematics 1327, Springer Verlag, Berlin Heidelberg New York, 1988.

3. L. Busé, Residual resultant over the projective plane and the implicitization problem, Proc. Annual ACM International Symposium on Symbolic and Algebraic Computing, B. Mourrain, editor, ACM Press, New York, 2001, pp. 48-55.

4. L. Busé, On the equations of the moving curve ideal, preprint arXiv:0712.2671 (2007).

5. L. Busé and M. Chardin, Implicitizing rational hypersurfaces using approximation complexes, Journal of Symbolic Computation 40 (2005), 1150-1168.

6. L. Busé and J.-P. Jouanolou, On the closed image of a rational map and the implicitization problem, J. Algebra (2003), 312-357.

7. D. Cox, Four conjectures: Two for the moving curve ideal and two for the Bezoutian, Proceedings of Commutative Algebra and its Interactions with Algebraic Geometry, CIRM, Luminy, France, May 2006, (available in CD media).

8. D. Cox, The moving curve ideal and the Rees algebra, Theoretical Computer Science 392 (2008), 23-36.

9. D. Cox, J. W. Hoffman, and H. Wang, Syzygies and the Rees algebra, J. Pure Appl. Algebra 212 (2008), no. 7, 1787-1796.

10. S.D. Cutkosky, J. Herzog and N.V. Trung, Asymptotic behaviour of the Castelnuovo-Mumford regularity, Compositio Math. 118 (1999), 243-261.

11. D. Eisenbud, Commutative Algebra with a view toward Algebraic Geometry, Graduate Texts in Mathematics 150, Springer Verlag, Berlin Heidelberg New York, 1995.

12. D. Eisenbud and J. Harris, Powers of ideals and fibers of morphisms, arXiv:0807.4243v1.

13. M. Elkadi and B. Mourrain, Residue and Implicitization Problem for Rational Surfaces, Applicable Algebra in Engineering, Communication and Computing 14 (2004), 361-379.

14. R. Hartshorne, Algebraic Geometry, Graduate Texts in Mathematics 52, Springer Verlag, Berlin Heidelberg New York, 1977.

15. M. Hochster, Properties of Noetherian rings stable under general grade reduction, Arch. Math. 24 (1973), 393-396.

16. J. Hong, A. Simis, W. V. Vasconcelos, On the homology of two-dimensional elimination, J. of Symbolic Computation 43 (2008), 275-292.

17. S. Huckaba and T. Marley, Depth formulas for certain graded rings associated to an ideal, Nagoya Math. J. 133 (1994), 57-69.

18. C. Huneke and B. Ulrich, General Hyperplane Sections of Algebraic Varieties, J. Algebraic Geometry 2 (1993), 487-505.

19. J.-P. Jouanolou, Résultant anisotrope, compléments et application. The Foata Festschrift, Research Paper 2, Electron. J. Combin, 3 (1996), approx. 91 pp.

20. J.-P. Jouanolou, Formes d'inertie et rsultant: un formulaire, Adv. Math. 126 (1997), 119250.

21. V. Kodiyalam, Asymptotic behaviour of Castelnuovo-Mumford regularity, Proc. Amer. Math. Soc. 128 (2000), 407-411. 
KPU-d 22. A. Kustin, C. Polini, and B. Ulrich, Divisors on rational normal scrolls, preprint (2008).

KPU-b 23. A. Kustin, C. Polini, and B. Ulrich, Blow-ups and fibers of morphisms, preprint (2008).

KPU-m 24. A. Kustin, C. Polini, and B. Ulrich, Equations of moving curves, preprint (2008).

L 25. L. Lipman, Cohen-Macaulayness in graded algebras, Math. Res. Lett. 1 (1994), 149-157.

SUV

26. A. Simis, B. Ulrich and W.V. Vasconcelos, Cohen-Macaulay Rees algebras and degrees of polynomial relations, Math. Ann. 301 (1995), 421-444.

T03 (2003), 99-113

TW

28. N. V. Trung and H.-J. Wang, On the asymptotic linearity of Castelnuovo-Mumford regularity, J. Pure Appl. Algebra 201 (2005), 42-48.

Mathematics Department, University of South Carolina, Columbia, SC 29208

E-mail address: kustin@math.sc.edu

Mathematics Department, University of Notre Dame, Notre Dame, IN 46556

E-mail address: cpolini@nd.edu

Department of Mathematics, Purdue University, West Lafayette, IN 47907

E-mail address: ulrich@math.purdue.edu 